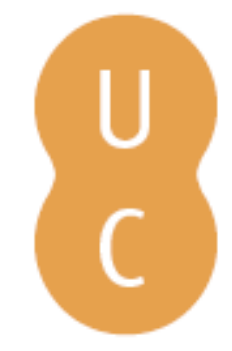

\title{
pommalina
}

\section{Le caractère cosmopolite et multiculturel des colonies grecques ouest-pontique}

\author{
Autor(es): $\quad$ Bechet, Florica
}

Publicado por: Imprensa da Universidade de Coimbra; Annablume

URL

persistente: $\quad$ URI:http://hdl.handle.net/10316.2/40841

DOI: $\quad$ DOI:https://doi.org/10.14195/978-989-26-1280-5_20

Accessed : $\quad$ 26-Apr-2023 16:22:08

A navegação consulta e descarregamento dos títulos inseridos nas Bibliotecas Digitais UC Digitalis, UC Pombalina e UC Impactum, pressupõem a aceitação plena e sem reservas dos Termos e Condições de Uso destas Bibliotecas Digitais, disponíveis em https://digitalis.uc.pt/pt-pt/termos.

Conforme exposto nos referidos Termos e Condições de Uso, o descarregamento de títulos de acesso restrito requer uma licença válida de autorização devendo o utilizador aceder ao(s) documento(s) a partir de um endereço de IP da instituição detentora da supramencionada licença.

Ao utilizador é apenas permitido o descarregamento para uso pessoal, pelo que o emprego do(s) título(s) descarregado(s) para outro fim, designadamente comercial, carece de autorização do respetivo autor ou editor da obra.

Na medida em que todas as obras da UC Digitalis se encontram protegidas pelo Código do Direito de Autor e Direitos Conexos e demais legislação aplicável, toda a cópia, parcial ou total, deste documento, nos casos em que é legalmente admitida, deverá conter ou fazer-se acompanhar por este aviso. 


\section{Pólis/Cosmópolis}

\section{Identidades Globais \& Locais}

Carmen Soares, Maria do Céu Fialho \& Thomas Figueira (coords.)

IMPRENSA DA UNIVERSIDADE DE COIMBRA 


\title{
LE CARACTÈRE COSMOPOLITE ET MULTICULTUREL DES COLONIES GRECQUES OUEST-PONTIQUE (The Cosmopolitan and Multicultural Character of the West-Pontic Greek Colonies)
}

\author{
Florica BeChet (floribecus@yahoo.com) \\ Université de Bucarest
}

Résumé - L'intention de notre article est de mettre en évidence les particularités cosmopolites et les principales directions culturelles qui distinguaient les colonies grecques, fondées par l'antique Milet et par Mégare ou par d'autres de ses colonies sur la rive ouest du Pont Euxin, sites archéologiques qui se trouvent de nos jours en Roumanie. Dans l'Antiquité, cette région était considérée, à la fois, un vrai Eldorado, grâce à ses richesses, mais aussi un paradigme de la sauvagerie, en comparaison avec la civilisation du monde grec. Dans notre démarche l'accent tombe sur deux éléments: d'un côté, les cultes religieux de la zone, de l'autre côté, les multiples aspects culturels manifestés dans ces colonies. On y trouve présentées les principales divinités cultivées dans cette région, qui sont, en premier lieu, les divinités des métropoles, identifiées par les épiclèses, mais aussi des dieux inconnues dans les métropoles, mais retrouvés dans d'autres colonies du basin de la Mer Noire; en deuxième lieu, il s'agit des divinités locales et des cultes qui leur sont attribués. Dans la partie dédiée à la culture nous présentons la situation de l'école et le niveau d'éducation des habitants, qui ont donné de personnalités importantes, y compris des écrivains, affirmés aux grandes cours grecques ou égyptiennes et dont les noms se sont transmis à la postérité. Nous mettons en évidence les connaissances en architecture et les tendances artistiques et sportives des habitants, la vie dans les gymnases et les théâtres, la vie des livres, qui étaient commercialisés dans la zone en quantités impressionnantes et qui bénéficiaient d'une telle importance qu'ils accompagnaient les bibliophiles même dans la tombe, la pratique de la médicine et de la philosophie. Les habitants des colonies semblent être toujours au courant avec les événements culturels des métropoles et de tout le monde grec, mais aussi avec les grandes découvertes scientifiques, comme le calcul du temps et de l'heure exacte. L'étude des inscriptions, surtout celles en vers, font preuve d'une bonne langue correctement utilisée, avec talent et imagination. Rien ne confirme la sauvagerie que la culte mais la hautaine Grèce attribuait à ce pays.

Mots-CLÉ: Mer Noire, colonie, religion, culture, cosmopolite

Aвstract - This paper sets out to highlight the cosmopolitan features and the main cultural trends which characterize these Greek colonies. They had been founded by ancient Miletos and Megara or by colonies of the latter on the western shore of the Pontos Euxeinos, and are now archaeological sites in Romania. In antiquity, this region was considered for its time a veritable Eldorado on account of its riches, but also a paradigm of wilderness, as compared to the civilization of the Hellenic world. The emphasis 
of my endeavour falls upon two elements: on the one hand, the local religious cults, on the other, various cultural aspects manifested in these colonies. I present the main deities worshiped in this area, which are, in the first place, the deities of the metropoleis, identified by their epikleseis, but also some deities that do not appear in the metropoleis, which the West Pontic settlements share with other colonies in the Black Sea basin. Secondly, there are the local deities and the cults that are attached to them. The part of the study that is reserved for culture focuses upon schooling and the educational level of the natives, among whom many notable people arose, including writers who became prominent at the great Greek or Egyptian courts and whose names have been handed down to posterity. The architectural knowledge and the artistic and athletic inclinations of the natives are highlighted, as well as life in gymnasia and in theatres; also the life of books, which used to be traded in impressive numbers in the region and were held in such esteem that they accompanied bibliophiles in their tombs; and finally the practice of medicine and philosophy. Nor only do the inhabitants of the colonies seem always to have been acquainted with the cultural events of the metropoleis and of the entire Greek world, but also with the great scientific discoveries, like those in calculating time. The study of inscriptions, especially of the ones in verse, reveals a correct usage of language, handled with knowledge, talent, and imagination. None of these characteristics confirms the savagery with which cultivated, but haughty, Greece labelled these lands.

KeYwords: Black Sea, colony, gods, culture, cosmopolite

Dès avant le VIII İ̀e s. a. J.-C., quand dans la littérature grecque apparaissent pour la première fois certaines mentions sur la zone pontique, le mental collectif grec percevait le nord traco-scythique, depuis les Carpates et la Mer Noire jusqu'à la Mer Caspienne, comme un vrais Eldorado. Une partie de cette zone, le littoral ouest-pontique, présentait d'incontestables avantages pour les étrangers venus de loin. Il s'agit, en premier lieu, d'excellentes conditions d'emplacement pour des installations portuaires, offerte à l'heure par les golfes. Ensuite, par une zone favorable pour l'agriculture et, vers l'intérieur du pays, par des collines couvertes de forêts et des prés fertiles, au moins une excellente source d'eau (celle de Fântânele), ainsi que des carrières de calcaire (les collines de Babadag) ou de cuivre (la mine d'Altân-Tepe). Evidement, ces étrangers se sont rendu compte du rôle que pourraient jouer leurs fondations pour le transport vers le monde égéo-méditerranéen des fruits de l'immense réservoir céréalier nord-danubien. Mais, malgré tous ces avantages et richesses, les habitants de ce pays représentaient un constant paradigme de la sauvagerie par rapport à l'humanisme civilisé du monde grec.

En même temps, les bords de la Mer Noire sont devenus, surtout après «la grande colonisation grecque» des VIIe-VIe siècles av. J.-C., un vrai empire milésien. Deux des ces colonies fondées par la ville de Milet, Histria/Histros (Constanța) et Tomi, se trouvent en Roumanie. On leur ajoute une localité située vers l'intérieur, peut-être dans la chora histriote, Orgamê, à une étroite relation avec Histros, et la dorienne Kallatis (Mangalia), colonie de la mégarienne Héraclée du Pont. 
Notre intention est d'examiner les cultes et la culture attestés dans ces colonies et les conclusions qui en dérivent du point de vue du caractère cosmopolite et multiculturelle de cette région.

\section{LeS CULTES LOCAUX}

Les riches données fournies notamment par les inscriptions provenant de ces colonies, mais également par les monnaies et par certains documents iconographiques permettent de classer les divinités attestées dans le panthéon de cette zone selon un schéma composé de divinités appartenant sûrement à la couche initiale des cultes célébrés par les colons arrivés, directement et pindirectement, de leurs métropoles grecques (Milet et Mégare, en passant par une première fondation de celle-ci, l'Héraclée du Pont),

Tout comme pour les institutions, la configuration originaire du panthéon de ces colonies a été déterminée par le «bagage initial» des colons.

\section{Apollon}

À Istros le culte fondamental était consacré à Apollon Iètros («Médecin»), un dieu à vocation de sauveur connu uniquement grâce à des documents milésiennes de la mer Noire: (Apollonia du Pont, Istros, Tyras, Olbia, Panticapéé, Hermonassa, Phanagoreia), mais pas à Milet. Il est bien possible qu'il s'agisse d'un ancien dieu ionien qui aurait disparu sans trop laisser de traces en Asie Mineure'; il s'agit d'un culte typique de génos aristocratique, dont le sacerdoce était monopolisé 2 par les descendants de l'oeciste.

À Istros le culte du «Médecin» est attesté indirectement à une époque très haute (première moitié du VIe s. av. J.-C.) grâce à la base de sa statue de culte, portant une dédicace datée du début du IVe s. av. J.-C. (ISM I 169) et à un graffite mentionnant le nom théophore Iètrodôros. L'éponyme de la cité était le prêtre d'Apollon Iètros (ISM I 7, 54, 63), et longtemps on a affaire à une vraie dynastie sacerdotale composée des successeurs du fondateur de la cité ${ }^{4}$ (ISM I $144,169,170)^{5}$. Distinctement d'Olbia, à Istros il n'y a jusqu'à l'heure aucun témoignage d'Apollon Delphinios. À l'époque romaine a pénétré à Istros le culte d'Apollon Phôleutèrios (ISM I 105: IIIe s. av. J.-C.) ${ }^{6}$, en directe relation avec la célèbre école de médicine fondé par Parménide.

${ }^{1}$ Ehrhardt 1989.

${ }^{2}$ Alexandrescu-Vianu 1988.

${ }^{3}$ Alexandrescu-Vianu 1990: 185-187 et fig. 3; Alexandrescu-Vianu 2000: cat. No 101 et pl. 43.

${ }^{4}$ Voir aussi les dédicaces ISM I 104 et $314 \mathrm{~A}$.

${ }^{5}$ Lambrino 1937; Alexandrescu-Vianu 1988.

${ }^{6}$ Cette épiclèse est un hapax. On a proposé d'en voir un adjectif tiré de pholeos (la célèbre école médicale fondé par Parménide) et d'expliquer l'introduction de ce culte par certains Éléates comme celui attesté à Callatis: Vinogradov 2000. 
De l'autre coté, le plus important des dieux mégariens est aussi Apollon, dont le culte est attesté également dans les colonies. Mais il n'y ait jusqu'à l'heure aucune inscription qui révèle l'épiclèse Pytios: car Apollon célébré à Mégare est par excellence celui de Delphes. Pourtant les monnaies l'attestent pleinement. De plus, les relations de cette ville avec l'oracle de Delphes sont illustrées par nombre de documents. En revanche, c'est une épiclèse plus rare, universellement dorienne plutôt que typiquement mégarienne, qui est attestée à Kallatis: Agyeus (ISM III 30); attestéé aussi à Tomi (ISM I 116 I, contamination avec le panthéon kallatien). À leur tour, les inscriptions oraculaires kallatiennes du IIe s. av. J.-C. font connaître des épithètes plus banales d'Apollon, comme Nomios (ISM III 48 $B$ et 49; Tropaeum Traiani d'Adamclisi).

\section{Artémis}

Les cultes consacrés à Artémis à Istros, dont on ne dispose de témoignages épigraphiques qu'à l'époque hellénistique (ISM I 172, 243, 256, 266) et de Léto (ISM I 170, du IVe s. av. J.-C.) se situent dans un rapport étroit avec le culte d'Apollon. À Kallatis Artémis est attesté par une épigramme funéraire du IIIe s. av. J.-C. pour une de ses prêtresses (ISM III 132) et par une biera trapéza que lui consacre au IIIe s. av. J.-C. une autre prêtresse (ISM III 32). Son culte est largement illustré par des documents divers à Mégare et dans ses colonies; à Kallatis il appartenait donc sans l'ombre d'un doute au bagage initial des colons ayant fondé la ville .

\section{Zeus}

La couche originaire du panthéon mis en place par les colons milésiens peut être complétée par le culte de Zeus, dont le temple (avec son bothros) et l'autel ont pu être identifiés grâce aux fouilles archéologiques dans la zone sacrée de la ville, mais aussi par un décret du IIIe s. av. J.-C.. L'épithète Polieus qui accompagne Zeus dans les inscriptions hellénistiques (ISM I 8, 54) et impériales (ISM I 222) témoignent du caractère civique plus tardif du culte. Cela dit, on serait assez proche de l'époque de l'apogée de l'influence d'Athènes, la patrie par excellence de Zeus Polieus, et éventuellement de la chute du régime oligarchique à Istros dont parle Aristote.

La relation entre Zeus et la famille d'Apollon est magistralement exprimée dans un beau relief daté du IIe s. av. J.-C. et représentant Apollon Citharède, Zeus et Artémis', ce qui a contribué à l'hypothèse d'une «famille de culte» du

${ }^{7}$ Hanell 1934: 134; Antonietti 1999.

${ }^{8}$ Sur le droit de certaines monnaies de bronze hellénistiques est figurée la tête d'Artémis: Pick 1898: no 248-253; cf. la contremarque au buste de la déesse avec arc et carquois, no 224a. À l'époque impériale, Artémis est représenté comme chasseresse sur le revers des monnaies de Septime Sévère (no 303), Iulia Mamaeaa (no 336), Philippe l’Arabe (no 351), Otacilia (no 363). Un beau relief d'époque hellénistique représente Artémis à la chasse: Preda, Popescu et Diaconu 1962: 450, fig. 10c; Bordenache 1960: 498 et fig. 10-11.

${ }^{9}$ S. Lambrino 1937 (qui date le relief du IVe s. av. J.-C.). Bordenache 1961: 201, fig. 16 
type attesté à Didymes, le sanctuaire proche de Milet (Apollon-Zeus-Léto) ${ }^{10}$.

À Kallatis aussi Zeus fait partie des dieux de la communauté civique et des magistrats (cf. le graffite ISM III 254, du début du IVe s. av. J.-C., qui est une dédicace à Zeus Sôtér). De l'époque hellénistique date un minuscule fragment d'inscription avec l'épiclèse Polieus (ISM III 22), et dans les inscriptions oraculaires du IIe s. av. J.-C. Zeus est attesté comme Hypatos (ISM III 48 B), Agoraios (?) et Hyperdexios (ISM III 49). Une épiclèse locale de Zeus,- Ombrimos («le pluvieux»), épiclèse homérique, utilisée seulement dans les sources littéraires, qui mélange de culte religieux et de culture littéraire, - pourrait être suggérée par les fêtes des Diombries (ISM III 31).

\section{Athéna}

La présence d'Athéna du panthéon milésien, avec sa «famille de culte» Apollon Delphinios-Zeus-Athéna, est discrète dans les documents épigraphiques d'Istros.

Par contre, son culte appartenant à la couche originaire du panthéon kallatien. À suivre Pausanias (I, 42, 4), la déesse était adorée à Mégare dans trois sanctuaires, consacrés respectivement à Athèna Aiantis, à Athéna Nikê et à une Athéna sans épithète, mais qui était sans aucun doute la Polias. Une Athéna Polias est connue à Kallatis dès le IVe s. av. J.-C. (ISM III 76). Elle est aussi Hypata et Hyperdexia dans les inscriptions oraculaires évoquées pour Zeus. À tout cela il convient d'ajouter les types iconographiques d'Athéna sur les monnaies kallatiennes ${ }^{11}$.

\section{Aphrodite}

Un autre culte important introduit par les premiers colons milésiens était consacré à Aphrodite. Les recherches archéologiques effectuées à Istros en ont identifié le temple. C'est toujours de l'époque archaïque que datent la dédicace figurant sur un calyptère (premier quart du VIe s. av. J.-C.: ISM I 101) et quelques terres-cuites représentant «la déesse à la colombe» ${ }^{12}$. Les autres documents concernant Aphrodite sont plus tardifs, comme un superbe relief (fin du IIe ou début du Ier s. av. J.-C.) représentant les noces d'Aphrodite avec Arès ou Adonis $^{13}$. L'iconographie de certaines terres-cuites d'époque archaïque renvoie au monde syrien: c'est peut-être la même Aphrodite syrienne que celle attestée par des graffites d'Olbia ${ }^{14}$.

= Bordenache 1969: cat. no 293 et pl. CXXVII; Alexandrescu-Vianu 2000:, cat. No 127 et pl. 57.

${ }^{10}$ Ehrhardt 1988: 121-161, 190.

${ }^{11}$ Pick 1898: no 234-247 et pl. I 24, 25 (époque hellénistique); 267-274, 309, 340 (époque impériale).

${ }^{12}$ Pippidi 1971; fig. 10-11.

${ }^{13}$ Alexandrescu-Vianu 2000: cat. no 124 et pl. 51-54.

${ }^{14}$ Alexandrescu-Vianu 1994, Alexandrescu-Vianu 1997. La seule épithète d'Aphrodite révélée par les inscriptions d'Istros est Pontia (ISM I 173). 
D'autre part, l'inscription oraculaire ISM III 49 et ISM III $48 \mathrm{~B}$ nous font part d'un groupe de divinités de l'agora kallatienne: Thémis Agoraia, Zeus, Aphrodite Agoraia ${ }^{15}$, Hermes Agoraios ${ }^{16}$, Apollon Apotropaios et Poseidon Asphaleus.

\section{Poséidon}

Pour continuer avec les dieux d'origine milésienne d'Istros, il convient de mentionner aussi Poséidon, adoré à Istros et à Tomi comme Hélikonios (ISM I 143, IIIe s. av. J.-C.) et notamment, à Istros, comme Taurios: les documents attestent, au IIe s. av. J.-C., une association de Tauréstai; (ISM I 60 et 61) ${ }^{17}$.

\section{Divinités agraires}

En ce qui concerne d'autres divinités, attestées à la fois à Istros, à Tomi et à Milet, sans pouvoir démontrer la filière milésienne, on peut citer Déméter Chtonia et Ga (ISM I 109, 115, 120, 125; ISM II 36 - la déesse tient l'éponymie divine de la cité: Ier s. av. J.-C.; ISM II 59, 118?, 150 - associée à Korè et à Plouton). Comme déesse agraire, Déméter semble avoir été très populaire dans le territoire, comme le preuve le sanctuaire extra-urbain de Nuntaşi, où Démeter est associée à Cybèle et à Nike ${ }^{18}$. À leur tour, Korê et Cybèle sont connues depuis d'époque archaïque tardive ${ }^{19}$.

Pour Kallatis une inscription de la fin de l'époque hellénistique nous renseigne sur l'existence d'une association cultuelle de thoinatai consacrée à Déméter Chtonia (ISM III 40), alors qu'une dédicace fragmentaire d'une autre association (d'époque impériale) est consacrée à Déméter Ploutodoteira (ISM III 259). D'autre part, Déméter est représentée sur des monnaies de bronze d'époque hellénistique ${ }^{20}$ de Tomi et de Kallatis.

C'est à Gé $(\mathrm{Ga})$ de compléter la liste des divinités chtoniennes kallatiennes: un sanctuaire en est mentionné dans l'inscription oraculaire ISM III $48 \mathrm{~B}$ du IIe s. av. J.-C., mais les origines du culte remontent sûrement à la fondation de la cité.

${ }^{15}$ À Kallatis, Aphrodite est connu comme Pandamos dès le IVe s. av. J.-C. dont le caractère est également éminemment civique. On peut ajouter un torse d'Aphrodite et d'Éros du I ${ }^{\text {er }}$ s. av. J.-C. (Bordenache 1069: 30-31, no 38) et une statue d'Aphrodite consacré par une prêtresse au IIIe s. ap. J.-C. (ISM III 94).

${ }^{16}$ La tête de Hermès est représentée sur des monnaies kallatiennes de bronze d'époque hellénistique : Pick 1898: no 253.

${ }^{17} \mathrm{Il}$ faut y ajouter une troisième inscription (fragmentaire) de la même époque, trouvé dans la zone sacrée et encore inédite, ainsi qu'un document plus tardif, du IIe s. ap. J.-C. (ISM I 57). Dans le calendrier de Histros on trouve le mois Taureon (ISM I 26) et une fête Taures est également connue (ISM I 60).

${ }^{18}$ Domăneanțu 1993.

${ }_{19}$ Alexandrescu-Vianu 1990; Alexandrescu-Vianu 1990. En Revanche, toutes les attestations épigraphiques de Cybèle (Meter aeon) datent de l'époque impériale :ISM I 57 (I, 16), 126-128.

${ }^{20}$ Pick 1898: nos 225-226 et pl I 22. Représentations de la tête de Déméter sur les monnaies de bronze d'époque impériale: nos 279-288. 
En ce qui concerne Kallatis, il convient tout d'abord de rattacher au groupe initial Dionysos Patrôos et Dasyllios, Aphrodite Pandamos, Peithô, Artémis, Déméter (Damater) Chthonia et Cronos. Le seul dieu qui n'appartient pas à cette catégorie d'anciennes divinités, bien qu'il figure dans la même liste, est Dionysos Bakkheus. Les épithètes Patrôos et Dasyllios de Dionysos sont exclusivement mégariennes, ce qui prouve que le culte a été introduit par les colons venus de Mégare à Héraclée du Pont et à partir dici à Kallatis. La plupart de ces divinités se retrouvent dans la description donnée par Pausanias (I 43, 5-6) du téménos de Mégare: il est donc à supposer que le téménos de Kallatis en fût une copie assez fidèle.

\section{Dionysos}

À Istros, les monnaies hellénistiques de bronze à la représentation de la tête de Dionysos ${ }^{21}$ mises à part, le culte du dieu de la vigne n'est attesté, par des inscriptions et par des monuments figurés, qu'à l'époque impériale ${ }^{22}$. Tout cela suggère une pénétration plus tardive de Dionysos dans le panthéon d'Istros.

Les documents de Tomi attestent des cultes de date récente, comme, par exemple celui de Dionysos Katêgémoneus «le dirigent» ou Pyribromos «l'étincelant».

Au contraire, à Kallatis le dieu bénéficiait de la plus riche documentation est: 11 inscriptions s'échelonnant depuis le IVe s. av. J.-C. jusque vers la fin du Ier s. ap. J.-C. La place occupée par Dionysos dans le panthéon de la cité est, d'autre part, mise en évidence par les monnaies hellénistiques de bronze à la représentation de Dionysos sur le droit ${ }^{23}$.

Les inscriptions révèlent trois épiclèses. La liste des divinités au IVe s. mentionne Dionysos Patrôos, Dionysos Bakkheus et (Dionysos) Dasyllios. Très importante s'avère la première des trois épiclèses; car il s'agit de la première attestation épigraphique de cette épiclèse adjointe à Dionysos, qui est confirmée par Pausanias $(\mathrm{I} 43,5)$ à Mégare. Le reflet presque parfait des cultes du Dionysos mégarien à Kallatis est également illustré par la troisième des épiclèses mentionnées, supposée par un sanctuaire désigné comme Dasyllieion. Cette épiclèse est, elle aussi, uniquement mégarienne, comme le prouvent Pausanias et Nonnos (Dionysiaques XXX 188-190).

L'épiclèse Bakkheus, attestée dès le IVe s. av. J.-C., se retrouve dans les dédicaces ISM III 79 et 80. D'autre part, le thiase dont on connaît plusieurs décrets est une fois désigné (ISM III 45) comme bakkbikos thiassos, alors que dans le règlement sacré (ISM III 47) figurent entre autres les néobakkhoi.

\footnotetext{
${ }^{21}$ Pick 1898: no 469, 470.
}

${ }^{22}$ Les inscriptions (ISM I 100, 142, 1167, 198, 203-206; 221; cf. SCIVA 50, 1999, 1-2, p. 71-77) mentionnent pour la plupart Dionysos Kepophoros. Parmi les reliefs, il n'y en a qu'un seul à dater éventuellement de l'époque hellénistique tardive: Alexandrescu-Vianu 2000: cat. No 173 et pl. 71.

${ }^{23}$ Pick 1898: nos 217-224 a et pl. I 20-21. 
Quelques fêtes consacrées à Dionysos sont connues d'une manière plus ou moins satisfaisante. Il s'agit tout d'abord des fêtes triétériques (ISM III 35 et 44) célébrées au mois Dionysios et les Dionysia ta xénika (ISM III 3 et 44), célébrées au mois Lykeios, qui sont les «fêtes où l'ont accomplit le xénismos de Dionysos».

Les données des inscriptions sur les édifices de culte consacrés à Dionysos complètent le tableau. Il s'agit, d'une part, du Dasyllieion, le sanctuaire de Dionysos Dasyllios, situé selon toute évidence à l'extérieur de la ville (ISM III 47), d'autre part, d'un temple désigné comme mykhos (ISM III 44) qui semble imiter une grotte dionysiaque prête à abriter des cérémonies accompagnées du mystères ${ }^{24}$.

\section{Autres divinités}

À côté des cultes d'origine milésienne ou transmises, plus ou moins probablement, par une filière milésienne, on découvre dans le panthéon d'Istros beaucoup d'autres divinités dont la pénétration peut être explicite par des influences venues d'ailleurs, notamment à l'époque hellénistique. Un culte qui n'est pas encore attesté à Milet mais qui était, selon toute évidence, très populaire sur la côte occidentale de la mer Noire est le culte consacré aux Dioscures, "chargés» de l'éponyme de la cité (ISM I 142) et vénérés comme «sauveurs» (ISM I 112). À Tomi, ils sont nommés Dioskorous ktistas poléos, ce qui représente le seul cas de ce type attesté dans le monde grec, mais qui peut plaider pour la fondation ou du moins une deuxième fondation de Tomi par Istros.

\section{Les Dieux de Samothrace}

Parmi les cultes introduits à l'époque hellénistique, il convient de réserver une place spéciale aux Dieux de Samothrace (voir en particulier ISMI 19), d'ailleurs très populaires dans tout le bassin de la mer Noire. À Istros est mentionné un Samothrakion (ISM I 11, 19, 58). La prêtrise des Dieux de Samothrace était publique (ISM I 19, I, 21-22) et va de pair avec l'intérêt porté par la cité, tout aussi que par Tomi (ISM II 1), aux relations avec le sanctuaire de Samothrace. Les mêmes dieux sont très en vogue à Kallatis, où ils ont d'un Samothrakion.

\section{Le Grand Dieu}

Une divinité tout à fait énigmatique est le «Grand Dieu» (Théos mégas), auquel un citoyen de Thasos dédie vers le milieu du IIIe s. av. J.-C. un temple (ISM I 145). Ce dieu n'a rien à voir avec le «Grand Dieu» Derzelas, un dieu d'origine thrace (le nom en prête serment), attesté à Odessos et dans ses environs par des inscriptions et des monnaies plus tardives; il n'est donc pas question, à Istros, d'un syncrétisme gréco-thrace.

\section{Divinités secondaires}

\section{Hérmès}

Quant à Hermès, son attestation comme Agoraios à Istros (ISM I 175 et 176) ne fait qu'enrichir le dossier déjà volumineux des divinités protectrices des

\footnotetext{
${ }^{24}$ Avram 1995 = 2002; Jaccottet 2003 II: 152-153.
} 
magistrats dans le monde hellénistique, alors que l'existence des fêtes Hermaia dans le gymnase (ISM I 59) est en état de confirmer le rôle du dieu dans ces lieux.

Dans le panthéon de Kallatis, c'est le héros Héraclès qui occupait une place de choix. Dans ce cas, le culte n'était pas mégarien, mais héracléote. Outre qu'il était le héros éponyme de la métropole, il n'est pas sans portée qu'Héraclès figurait à partir du IVe s. av. J.-C. sur les premières émissions monétaires d'argent de Kallatis. D'autre part, le premier sanctuaire attesté à Kallatis est celui de Héraclès (ISM III 3). Une éventuelle origine béotienne, filtrée par Mégare, pourrait être suggérée par l'épithète Aléxikakos porté par le héros dans une dédicace des thoinâtai du Ier s. ap. J.-C. (ISM III 68).

\section{Asclépios et ses conjoints}

L'époque hellénistique offre à Istros les premiers témoignages des cultes consacrés à Asclépios (ISM I 124: IIIe s. av. J.-C.).

À Kallatis plusieurs divinités sont documentées d'une manière indirecte: Asclépios (?)25 et Hygie $^{26}$ (ISM III $48 \mathrm{~B}$, mais aussi à Tomi ISM II 117), les Nymphes accompagnées de Pan (?), d'Hermès (?) et de Dionysos (?) dans un ensemble de divinités pastorales dans la même inscription.

Cronos appartient à un groupe d'anciennes divinités chtoniennes, qui comprend également Déméter Chthonia.

\section{Divinités marines}

Comme il est naturel dans un pays de marins et de commerçants, les épigraphes et les représentations figurées prouvent l'existence d'un culte de plusieurs divinités marines mineures, comme Phorkys. On ne peut pas négliger le dieu Pontos, représenté aux cotés de la déesse Fortuna dans une fameuse sculpture.

\section{Divinités étrangères}

On constate une pénétration des cultes orientaux, avec Cybèle en tête de file, représentée à Kallatis par quelques reliefs de marbre ou de terre cuite et par des représentations monétaires. Elle figure parfois associée au Cavalier Thrace. Attis est, à son tour, documenté par des terres cuites d'époque hellénistique.

C'est toujours vers le début de l'époque hellénistique que l'on voit les cultes égyptiens pénétrer en mer Noire. Il est peut-être utile de rappeler qu'un Alexandrin est attesté à Kallatis au IIIe s. av. J.-C. (ISM III 155), qu'un Kallatien figure comme mercenaire dans l'armée d'Alexandre le Grand et que des intellectuels d'origine kallatienne fréquentent les milieux alexandrins et s'établissent même à Alexandrie. C'est, non pas en dernière ligne, grâce à de tels personnages que les cultes égyptiens (Sarapis, Isis et Anoubis) se propagèrent dans les villes pontiques.

\footnotetext{
${ }^{25}$ Représentation sur des bronzes d'époque impériale : Pick 1898; no 352.

${ }^{26}$ Représentations sur des bronzes d'époque impériale: Pick 1898: nos 330 et 353.
} 
Le Cavalier Thrace est en vogue, comme à peu près partout dans la région balkanique, à partir de la basse époque hellénistique, mais surtout à l'époque impériale.

\section{Les abstractions}

Il y a enfin un groupe de cultes dont il est permis de supposer, au moins dans l'état actuel de nos connaissances, qu'ils aient été introduits à l'époque impériale plutôt qu'à une date plus ancienne.

Un exemple en est Némésis, dont la statue consacrée par les magistrats («archontes») après le milieu du IIIe s. ap. J.-C. (ISM III 75) est l'un des documents épigraphiques à témoigner de son culte.. À part les dieux et les déesses, le répertoire kallatien compte ensuite quelques abstractions divinisées. Il s'agit de Peithô (la Persuasion), attestés dès le IVe s. (ISM III 48 A). Il convient d'ajouter Agatos Daimôn, qui tient l'éponymie à la date du décret ISM III 38 du Ier s. av. J.-C., Agatê Tyykhê et la Concorde - Omonoïa (ISM III 1 et 41).

C'est toujours à une époque plus tardive, plus exactement à partir de la fin du IIIe s. ap. J.-C., que sur certaines monnaies locales de bronze figure une personnification de la ville de Kallatis.

À Istros, la communauté civique entretenait un culte du «Peuple» personnifié (Dêmos) dont une statue était exposée dans l'agora (ISM I 19). Dans le temple d'Aphrodite était déposée la triade des Moïraï.

\section{La culture locale}

L'impression générale qui résulte de l'analyse des documents est celle d'une culture provinciale, évidemment plus modeste par rapport aux grands centres de l'époque, mais pas moins hellène dans son ensemble.

Les plus riches informations se réfèrent aux divers aspects de la vie du gymnase. Le gymnase d'Istros, avec ses gymnasiarches, est bien connu depuis le IIIe s. av. J.-C. (ISMI 44, 59, etc.). Nous trouvons des informations sur les fêtes célébrées ici, surtout pour Hermès et Héraclès, divinités par excellence protectrices du gymnase. Une inscription de l'époque impériale montre les nombreuses bienfaits d'une dame Aba, la fille du notable Hekataios, adressées aux diverses associations, entre autre celle de professeurs, paideutai. Nous avons aussi des renseignements sur le gymnase et les gymnasiarches de Tomi et de Kallatis,. Les inscriptions parlent d'un paidotribas (ISM III 16) et d'un didaskalos doté de la qualité de proxène de Messambria pour ses mérites didactiques (ISB I2 307bis).

Les inscriptions parlent d'un théâtre à Istros et à Kallatis, mais en existait-il peut-être aussi à Tomi.

Au plaisir d'une élite istrienne de cultiver les lettres et les arts a répondu, au IIIe s. av. J.-C., un bienfaiteur local, Diogène, fils de Glaukias, qui a consacré un lieu de culte aux Muses (Mouseion), dont la prêtrise lui a été accordée de façon héréditaire, toute la famille ayant la grâce de grades honneurs publiques.

Il faut tenir compte du fait que le commerce a favorisé non seulement la circulation des marchandises, mais aussi celle des idées. On est certain que les 
écritures les plus importantes pénétraient dans les milieux grecs pontiques (voir une mention de Xénophon, Anabasis VII, 5, 14, qui avait vu à Salmydessos «beaucoup de rouleaux de papyrus écrits» parmi les débris des épaves de navires venues du Pont Euxin). Nous avons la première preuve du commerce des livres dans l'Antiquité, preuve confirmé par une découverte d'importance exceptionnelle à Kallatis: «la tombe à papyrus» (IVe s. av. J.-C.) - tombe tumulaire à ciste de pierre, où se trouve le squelette d'un personnage qui tient dans sa main droite un papyrus. Il y a aussi beaucoup de reliefs funéraires des maîtres d'école représentés avec leurs élèves qui, eux aussi ont un rouleau de papyrus.

Comme l'enseignement local ne suffisait pas, beaucoup de jeunes gens partaient à l'étranger, pour continuer leurs études dans les grands centres du monde hellénistique.

D'autre part, les villes de Doubroudja profitaient des séjours, plus ou moins longs, des professionnels qui donnaient des conférences (akroaseis), comme un médecin de Cyzique, honoré par un décret d'Istros (ISM I 26). Le culte d'Apollon Pholeutrios dans la même cité (ISM I 105) fait probable la présence dans la zone des médecins éléates (comme un citoyen d'Élée mentionné à Kallatis (ISM III 8) ou celle des citoyens du Pont qui effectuaient des stages à la célèbre école fondée par Parménide (phôléos). C'est entre autres grâce à la qualité de son enseignement que la ville de Kallatis a pu produire quelques historiens; écrivains et philosophes qui allaient acquérir une certaine renommée parmi leur contemporains et dont les activités ont parfois été retenues même par la postérité.

Le niveau de culture dans les colonies ouest-pontiques peut être apprécié satisfaisant, même remarquable, grâce aux textes conservés par les inscriptions. Bien qu'ils gardent des éléments dialectaux ioniques (jusque vers le milieu du IVe s. av. J. C.), respectivement, à Kallatis, des éléments du dialecte dorique, jusque vers les premières années de l'ère chrétienne, passant ensuite à la kolvń, les inscriptions offrent des textes non seulement corrects, mais parfois même d'une rédaction élégante. Par exemple, les décrets d'Istros (ISM I 15 ou 64) présentent une narration comparable, du point de vue stylistique, aux ouvres historiques de l'époque hellénistique. Les épigrammes funéraires sont des productions littéraires remarquables, qui usent avec grand ingénuité des thèmes communs dans le domaine; elles sont très correctes - à l'exception de très peu d'exemples tardifs - du point de vue métrique. La présence même de ces thèmes dans ce coin «sauvage» du monde antique est une preuve de plus de la circulation des idées et de l'intégration de la zone en question dans cette circulation.

La ville de Kallatis a donné même des gens de lettres. La postérité a gardé la mémoire de quelques lettrés dont les œuvres se sont perdues. Il s'agit de Démétrios, originaire d'Odessos, mais connu comme Démétrios de Kallatis, grâce au fait qu'il était devenu citoyen de cette ville (dans la deuxième moitié du IIIe s.). Son œuvre en 20 livres, Sur l'Europe et sur l'Asie, mentionnée par Diogène Laërce dans les Vitae (V 83) a été utilisée par Pseudo-Skymnos et louée expressis uerbis 
(v. 718-720) comme une autorité incontestable en questions d'histoire locale.

Il y a aussi Hérakléidès, le fils de Sarapion (surnommé Lembos), un Kallatien du IIe s. av. J. C., qui a fait une carrière notable en Egypte dans la suite du roi Ptolémée le VIe Philométor (Diogène Laërce, Vitae V 94; cf. VIII 7; 44; 58), l'auteur d'un impressionnant nombre de biographies et de compilations de philosophie.

En troisième lieu, il y a Istros de Kallatis qui, d'après Stéphane de Byzance (Kallatis s. v.) avait écrit «un beau livre sur la tragédie». Diogène Laërce (Vitae I 38) mentionne aussi un rhéteur Thalès.

Il paraît que même les sciences exactes n'étaient pas négligées dans ces colonies. Les Grecs des colonies mesuraient le temps de la même façon que ceux de la métropole. Nous savons qu'ils avaient gardé, par exemple, les mêmes mois de l'année qu'à Milet ou à Mégare. Donc l'année était coupée de la même façon et marquée par les mêmes fêtes. Pour mesurer le temps d'un jour on utilisait, tout comme les gens de la métropole, les cadrans solaires. À Istros et dans un village de la chôra tomitaine les archéologues ont découvert deux cadrans solaires de facture milésienne. Mais, chose d'une importance exceptionnelle, ces objets n'étaient pas importés de Milet. Les istriens s'étaient rendu compte qu'un cadran solaire de Milet n'étant d'aucune utilité à Istros. Alors ils se sont procuré un instrument très précis, parfaitement adapté aux coordonnés de leur villes. C'est le savant Constantin Popescu Cârligel qui, par des impressionnants calcules, a démontré les particularités du cadran d'Istros. Les historiens avaient peut-être envoyé à Milet des compatriotes qui apprennent l'art du calcul et de la construction des cadrans solaires ou, chose moins probable, ils ont fait venir un spécialiste de Milet (il y a quand même deux cadrans).

Donc, comme on peut voire, même s'ils gardaient leur ancien bagage religieux, ces «sauvages» des colonies ouest-pontiques accordaient leurs montres à toutes les clepsydres de leur temps. 


\section{Bibliografia Final}

AA.VV. (1990), Archéologie de la vigne et du vin. Actes du colloque 28-29 mai 1988, Paris.

AA.VV. (1992), Archeologia del paesaggio. IV Ciclo di lezioni sulla ricerca applicata in archeologia, Certosa di Pontignano (Siena) 14 - 26 gennaio 1991, Firenze.

AA.VV. (1997), Uomo, acqua e paesaggio. Atti dell'incontro di studio sul tema irreggimentazione delle acque e trasformazione del paesaggio antico, S. Maria Capua Vetere 22 - 23 novembre 1996, Roma.

AA.VV. (1998), El vi a l'antiguitat. Economia, producció i comerç al Mediterrani occidental. II Colloqui internacional d'arqueologia romana. Actes. Badalona, 6 - 9 de maig de 1998, Badalona.

AA.VV. (1999), El vino en la antigüedad romana. Simposio arqueología del vino, Jérez 2, 3 y 4 de octubre 1996, Madrid.

AA.VV. (1999b), Environmental reconstruction in Mediterranean landscape archaeology, Oxford.

AA.VV. (2001), La cerveza en la antigüedad, Sevilla.

AA.VV. (2004), Le vin. Nectar des dieux, génie des hommes, Gollion.

Abascal, J. Manuel, Espinosa, Urbano (1989), La ciudad hispano-romana. Privilegio y poder, Logronho.

Abásolo, J. A., Mayer, M. (1997), "Inscripciones latinas”, in S. Corchón (coord.), La Cueva de la Griega de Pedraza (Segovia), Zamora, 183-259.

Abbondanza, L. (ed.) (2008), Filostrato Maggiore, Milano.

Acosta-Hughes, B. (2002), Polyeideia. The Iambi of Callimachus and the Archaic Iambic Tradition, Berkeley and Los Angeles.

Adams, C. (2001), "There and back again. Getting around in Roman Egypt", in Adams, C. and R. Laurence (eds.), Travel and Geography in the Roman Empire, Londres and Nova Iorque, 138-166.

Adams, J. N. (2003), Bilingualism and the Latin language, Cambridge.

Adams, J. N. (2003a), “Romanitas and the Latin language”, CQ 53: 184-205.

Affatato, R. (2010), "Nueva York: recepción del mito de la ciudad en Federico García Lorca e Italo Calvino", in J. M. Losada Goya (ed.), Mito y mundo contemporáneo. La recepción de los mitos antiguos, medievales y modernos en la literatura contemporánea, Bari, 627-640.

Albuquerque, M. de (1968), O poder politico no Renascimento português, Lisboa.

Albuquerque, M. de (1981), "Bártolo e bartolismo na história do direito português”, Boletim do Ministério da Justiça 304: 41-61.

Albuquerque, M. de (1983), Estudos de cultura portuguesa, I, Lisboa. 
Alexandrescu-Vianu, M. (1988), “O nouă posibilă genealogie a familiei lui Hippolochos, fiul lui Theodotod, de la Histria”, SCIVA 39.3: 275-280.

Alexandrescu-Vianu, M. (1989), "Apollon Ietros. Ein verschollener Gott Ioniens?”, IstMitt 39: 115-122.

Alexandrescu-Vianu, M. (1990), "Die Steinskulptur von Histria", in P. Alexandrescu, W. Schuller (eds.) Histria. Eine Griechenstadt an der rumänischen Schwarzmeerkünste, Xenia. Konstanzer Althistorische Vorträge und Forschungen 25, Konstanz, 179-232.

Alexandrescu-Vianu, M. (2000), "Une alternative d'identification de la statue colossale d'Istros", in A. Avram, M. Babeş (eds.) Civilsation grecque et cultures antiques périphériques. Hommages à $P$. Alexandrescu à son $70^{\circ}$ anniversaire, Bucarest, 274-281.

Alexandridis, A. (2004), Die Frauen des römischen Kaiserhauses. Eine Untersuchung ibrer bildlichen Darstellung von Livia bis Iulia Domna, Mainz.

Alfayé, S., Marco, F. (2008), "Religion, language and identity in Hispania: Celtiberian and Lusitanian rock inscriptions", in R. Häußler (ed.), Romanisation et épigraphie. Etudes interdisciplinaires sur lacculturation et lidentité dans l,Empire romain, Montagne.

Alföldi, A. (1948), The conversion of Constantine and Pagan Rome, Oxford.

Alföldy, G. (1969), Fasti Hispanienses, Wiesbaden.

Alföldy, G. (1973), Flamines provinciae Hispaniae citerioris, Madrid.

Alföldy, G. (1991), "Augustus und die Inschriften: Tradition und Innovation. Die Geburt der imperialen Epigraphik”, Gymnasium 98: 289-324.

Allen, A. (1951), History of political thought in the sixteenth century. London

Altaner, B., Stuiber, A. (2a ed. 1972), Patrologia, São Paulo.

Amouretti, M.C., Brun J.-P. (eds.) (1993), La production du vin et de l'buile en Méditerranée. Actes du symposium international organisé par le Centre Camille Jullian et le Centre archéologique du Var, Aix-en-Provence et Toulon 20-22 novembre 1991 (BCH suppl. 26), Athènes.

Ando, C. (2003), "A Religion for the Empire", in A. J. Boyle, W. J. Dominik (eds.), Flavian Rome. Culture, Image, Text, Leiden, Boston 323-344.

Ando, C. (2006), "Interpretatio Romana”, in L. de Blois, P. Funke, J. Hahn, (eds.), The Impact of Imperial Rome on Religions, Ritual and Religious Life in the Roman Empire, Proceedings of the Fifth Workshop of the International Network Impact of Empire (Roman Empire 200 B.C. - A.D. 476.), Leiden, Boston 51-65.

Andrade, A. A. (1959), S. Tomás de Aquino no periodo áureo da filosofia portuguesa, Lisboa.

Andrade, A. A. de (1965), Antologia do pensamento politico português (séc. XVI), vol. I. Lisboa. 
Andrade, M. (1974), "Lira Paulistana”, in Poesias completas, São Paulo.

Andreu, J. (2004), Edictum, Municipium y Lex: Hispania en época flavia (69-96 d. C.), BAR Int. Ser. 1293, Oxford.

Antonietti, C. (1999), "Megara e le sue colonie: unità storico-culturale?”, in C. Antonetti, P. Lévêque (eds.) Il dinamismo della colonizzazione greca, Atti della tavola rotonda "Espansione e colonizzazione greca di età arcaica: metodologie e problemi a confront", Venezia, 10-11/11, Besançon-Paris, 17-24.

Aquino, T. de (1946), Des lois de Saint Thomas d'Aquin. Texte traduit et présenté par J. de la Croix Kaelin O. P., Paris.

Arnaldi, A. (2010), “Osservazioni sul flaminato dei Divi nelle provincie africane”, in M. Milanese, P. Ruggeri, C. Vismara, (eds.), L'Africa romana. Luoghi e le forme dei mestieri e della produzione nelle provincie africane. Atti del XVIII convegno di studio. Olbia 11-14 dicembre 2008, vol. III, Roma, 1645-1665.

Arruda, A. M. (2005), "O 10 milénio a.n.e. no Centro e no Sul de Portugal: leituras possíveis no início de um novo século", O Arqueólogo Português Série IV: 23: 59-74.

Arzone, A. (2011), “Alcune considerazioni sulle immagini di pietre miliari e sui riferimenti alle strade nel documento monetale", in I miliari lungo le strade dell'Impero. Caselle di Somma campagna Verona, 77-92.

Asensi, R. M., Musso, O. (1990), "Un documento etrusco di Tarragona", Quaderni della sezione di Studi Storici Alberto Boscolo 1: 5-11.

Aston, M. (1997), Interpreting the landscape. Landscape archaeology and local bistory, London.

$A T L=$ B.D. Meritt, WadeGery, H.T., McGregor, M.F., The Athenian Tribute Lists, 4 vs, Princeton.

Aubert, J.-M. (1955), Le droit romain dans l'oeuvre de Saint Thomas, Paris.

Avery, H. C. (1971), "Euripides' Heraclidae", AJPh 92: 539-565.

Avram, A., Lefèvre, F. (1995), "Les cultes de Callatis et l'oracle de Delphes", REG 108: 7-23.

Bacchielli, L. (1986), "Monumenti funerari a forma di cupula: origine e diffusione in Italia meridionale", in A. Mastino (ed.), L' Africa Romana: atti del 3. convegno di studio Sassari 13-15 dicembre 1985, Sassari, 303-319.

Bailly, A. (1963), Dictionnaire grec-français, Paris.

Balass, G. (s.d.), "The Female Breast as a Source of Charity: Artistic Depictions of Caritas Romana", www.Academia.edu/4006836.

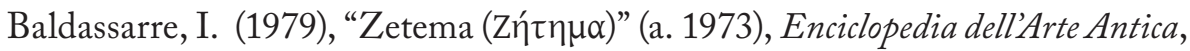
Suppl. 1979: 944-945.

Baldassarre, I., Bragantini, I., Morselli, C. and Taglietti, F. (1996), Necropoli di Porto. Isola Sacra, Roma. 
Balil, A. (1984-88), "Las cupae de Barcino. Contribución al estudio de un tipo de monumento funerario romano", Arqueologia e Historia: 111-115.

Baratta, G. (1993), “Una divinità gallo-romana. Sucellus. Un’ipotesi interpretativa”, ArchCl 45: 233-247.

Baratta, G. (1994), "Circa Alpes ligneis vasis condunt circulisque cingunt", ArchClass 46: 232-260.

Baratta, G. (1997), "Le botti: dati e questioni”, in Techniques et économie antique et médiévale. Le temps de l'innovation. Colloque international, Aix-enProvence 21-23 Mai 1997, Paris, 109-112.

Baratta, G. (1997), "Sucellus", in Enciclopedia dell'Arte Antica classica e orientale, Supplemento 1991-1994, V, Roma, 482.

Baratta, G. (2005a), "La cupa nell'ambito femminile: dalla caupona al loculus?”, in, F. Cenerini, A. Buonopane (eds.), Donna e vita cittadina nella documentazione epigrafica, 95-108.

Baratta, G. (2005b), Römische Kelteranlagen auf der italienischen Halbinsel. Ein Überblick über die schriftlichen, bildlichen und archäologischen Quellen (200 v.Chr. - 400. n.Chr.) (Cornucopia, 11), Murcia.

Baratta, G. (2005c), “Appunti sulle variabili e costanti dell' interpretatio religiosa nell'occidente romano, in F. de Oliveira, (ed.), Génese e consolidação da Ideia de Europa, vol.III, O Mundo Romano, Coimbra, 123-134

Baratta, G. (2006a), "Alcune osservazioni sulla genesi e la diffusione delle cupae", in Atti del XVI Convegno internazionale de L'Africa Romana (Rabat, 15-19 dicembre 2004), Roma, 355-368.

Baratta, G. (2006b), "Nuovi dati sull'iconografia delle mandorle nei sarcofagi strigilati. Un primo approccio ad un corpus", Annali della Facoltà di Lettere e Filosofia dell'Università di Macerata 26: 65-120.

Baratta, G. (2007), "La mandorla centrale dei sarcofagi strigilati. Un campo iconografico ed i suoi simboli", in F. Hoelscher, T. Hoelscher (eds.), Römische Bilderwelten. Von der Wirklichkeit zum Bild und zurück. Kolloquium der Gerda Henkel Stiftung am Deutschen Archäologischen Institut Rom, Heidelberg, 191-215.

Baron, H. (1938), "Cicero and the Roman civic spirit in the Middle Ages and the Early Renaissance", Bulletin of the John Rylands Library 22: 84-89.

Baron, H. (1970), La crisi del primo Rinascimento italiano, Firenze.

Barresi, P. (2007), "Il sofista Flavio Damiano di Efeso e la costruzione di termeginnasi nell'Asia Minore romana di età imperiale", in O. D. Cordovana, M. Galli, (eds.), Arte e memoria culturale nell'età della Seconda Sofistica, Catania, 137-151.

Barros, J. de (1919), Geografia d'Entre Douro e Minho e Trás-os-Montes, Porto. 
Barros, J. de (1937), Panegíricos - Panegírico de D. João III e da Infanta D. Maria, Texto restituído, prefácio e notas por M. Rodrigues Lapa, Lisboa.

Bassignano, M.S. (1974), I faminato nelle provincie romane dell'Africa, Roma.

Bastos, E. (1991), Entre o escândalo e o sucesso. A semana de 22 e o Armory show, Campinas.

Battaglia, M. (2003), "Il Vulcano dei Germani in Giulio Cesare (B.G. VI, 21, 1). Un caso di interpretatio?", Athenaeum 91: 373-401.

Beagon, M. (2005), The Elder Pliny on the Human Animal: Natural History Book 7, Oxford.

Beard, M., North, J., Price, S. (1998), Religions of Rome, vol. I, A History, Cambridge.

Behr, C.A. (ed.) (1973), Aristides, vol. I, Panathenaic Oration in Defence of Oratory, London.

Behrends, M. et alii (eds.) (2000), Hygin. L'oeuvre gromatique, Luxemburg.

Bejarano Osorio, A. M. (1996), "Sepulturas de incineración en la necrópolis oriental de Mérida: las variantes de cupae monolíticas", Anas 9: 37-58.

Belmonte, J. A. (2010), "Documentación fenicio-púnica en la Península Ibérica: estado de la cuestión", in G. Carrasco y J. C. Oliva (eds.), El Mediterráneo antiguo: lenguas y escrituras, Cuenca, 159-220.

Beltrán, F. ed. (1995), Roma y el nacimiento de la cultura epigráfica en occidente, Zaragoza.

Beltrán, F. (2000), "La vida en la frontera", in F. Beltrán, M. Martín-Bueno y F. Pina, Roma en la Cuenca Media del Ebro. La romanización en Aragón, Zaragoza.

Beltrán, F. (2002), "Identidad cívica y adhesión al príncipe en las emisiones municipales hispanas", in F. Marco, F. Pina y J. Remesal (eds.), Religión y propaganda politica en el mundo romano, Barcelona, 159-187.

Beltrán, F. (2004), "E1 latín en la Hispania romana: una perspectiva histórica", in R. Cano (ed.), Historia de la lengua española, Barcelona, 83-106.

Beltrán, F. (2004a), "Nos Celtis genitos et ex Hiberis. Apuntes sobre las identidades colectivas en Celtiberia", in G. Cruz Andreotti y B. Mora Serrano (eds.), Identidades étnicas - Identidades políticas en el mundo prerromano hispano, Kronion 1, Málaga, 87-145.

Beltrán, F. (2004b), “De nuevo sobre la tésera Froehner", Palaeohispanica 4: 4565.

Beltrán, F. (2004c), "Imagen y escritura en la moneda hispánica", in F. Chaves y F. J. García (eds.), Moneta qua scripta. La moneda como soporte de la escritura. Actas del III Encuentro Peninsular de Numismática Antigua, Anejos de AEspA 33: 125-139. 
Beltrán, F. (2004d), "Libertos y cultura epigráfica en la Hispania republicana", in F. Marco, F. Pina y J. Remesal (eds.), Vivir en tierra extraña: emigración e integración cultural en el mundo antiguo, Barcelona, 151-175.

Beltrán, F. (2005), "Cultura escrita, epigrafía y ciudad en el ámbito paleohispánico", Palaeohispanica 5: 21-56.

Beltrán, F. (2006), "Hispania y el Mediterráneo en los siglos II y I a. E.: diversidad cultural y movilidad social", in F. de Oliveira, P. Thiercy, R. Vilaça (eds.), O mar greco-latino, Coimbra, 223-240.

Beltrán, F. (2009), "Vltra eos palos. Una nueva lectura de la línea 7 de la Tabula Contrebiensis", in Espacios, usos y formas de la epigrafía hispana en épocas antigua y tardoantigua. Homenaje al Dr. Armin U. Stylow, Anejos de AEspA 48: 33-42.

Beltrán, F. (2011), "Lengua e identidad en la Hispania romana”, Palaeohispanica 11:19-59.

Beltrán, F. (2011a), “Firmas de artesano o sedes de asociaciones comerciales? A propósito de los epígrafes musivos de Caminreal (E.7.1), Andelo (K.28.1) y E1 Burgo de Ebro (HEp 11, 2001, 621 = AE 2001, 1237)", in E. Luján y J. M. García Alonso (eds.), A Greek man in the Iberian street. Papers in Linguistics and Epigraphy in honour of Javier de Hoz. Innsbrucker Beiträge zur Sprachwissenschaft 140, Innsbruck, 139-147.

Beltrán, F. (2011b), “Les colonies latines d'Hispanie (IIe siècle av. E.): émigration italique et intégration politique", in N. Barrandon y F. Kirbihler (eds.), Les gouverneurs et les provinciaux sous la République romaine, Rennes, 131-144.

Beltrán, F. (2012), "Roma y la epigrafía ibérica sobre piedra del nordeste peninsular", Palaeohispanica 12: 9-30.

Beltrán, F. (inédito), "Diversidad cultural y epigrafía: el ejemplo de Hispania", XII Congressus Internationalis epigraphiae Graecae et Latinae, Barcelona septiembre de 2002.

Beltrán, F., Estarán, M. J. (2011), “Comunicación epigráfica e inscripciones bilingües en la Península Ibérica”, in C. Ruiz Darasse y E. Luján (eds.), Contacts lingüistiques dans l'Occident méditerranéen antique. Collection de la Casa de Velázquez (126), Madrid, 9-25.

Beltrán, F., Velaza, J. (2009), "De etnias y monedas: las "cecas vasconas", una revisión crítica", in J. Andreu (ed.), Los vascones de las fuentes antiguas: en torno a una etnia de la antigüedad peninsular, Barcelona, 99-126.

Beltrán, F., Arasa, F. (1979-1980), "Los itineraria privata en la epigrafía latina”, Historia Antiqua, 9-10: 7-29.

Beltrán, F., Jordán, C., Marco, F. (2005), "Novedades epigráficas en Peñalba de Villastar (Teruel)", Palaeohispanica 5: 911-956.

Bentley, J. H. (1978), Politics and culture in Renaissance Naples, Princeton. 
Berciu, I.,Wolski, W. (1970), "Un nouveau type de tombe mise au jour à Apulum et le problème des sarcophages à voûte de l'Empire romain”, Latomus 29: 919-965.

Bergmann, M. (1998), Die Strablen der Herrscher. Theomorphes und politische Symbolik im Hellenismus und in der römischen Kaiserzeit, Mainz.

Berruti, V., Magistà, A. (eds.) (2009), L'automobile. Marche e modelli dalle origini a oggi, vol. 6, Lancia, Roma.

Besnier M., Chapot, V. (1913), "Via”, Dictionnaire des Antiquités Grecques et Romaines, 5, Paris, 777-817.

Bettini, C. (2008), “Tre Valascos nell'Italia del quatrocento: Meser Valasco di Vespasiano da Bisticci, Petrus Vallascis di Cataldo Siculo e Vasco Fernandes de Lucena", Humanitas 60: 205-226.

Bettini, M., Boldrini, M., Calabrese, O., Piccinni, G. (eds.) (2010), Miti di città, Siena.

Binsfeld, W. (1979), "Zu treverischen Kultdenkmälern”, in Festschrift 100 Jahre Rheinisches Landesmuseum Trier. Beiträge zur Archäologie und Kunst des Trierer Landes, Mainz, 263-269.

Blackman, D. (1969), "The Athenian Navy and Allied Naval Contributions in the Pentecontaetia", GRBS 10: 179-216.

Blanco Freijeiro, A. (1977), El puente de Alcántara en su contexto historico, Madrid.

Boardman, J. (1986), I Greci sui Mari. Traffici e Colonie, Trad. ital., Firenze, Giunti.

Boffo, L. (1975), "Cimone e gli alleatidi Atene”, RIL 109: 442-50.

Bol, R. (1984). Das Statuen programm des Herodes-Atticus-Nymphäums, Berlin.

Bona, G. (ed.) (1988), Pindaro. I peani, Cuneo.

Bonfante, G., Bonfante, L. (2002), The Etruscan language. An introduction. Revised edition, Manchester and New York.

Bonneville, J.-N. (1981), "Les cupae de Barcelone: les origines du type monumental", $M C V 17$ : 5-38.

Bontems, C. (1965), Le prince dans la France des XVIe e XVIIe siècles, Paris.

Bordenache, G. (1960), "Antichità greche e romane nel nuovo Museo di Mangalia”, Dacia N. S. 4: 489-509.

Bordenache, G. (1961), "Histria alla luce del suo materiale scultureo”, Dacia N. S., 185-211.fig. 16.

Bordenache, G. (1969), Sculture greche e romane del Museo Nazionale di Antichità di Bucarest I. Statue e rilievi di culto, elementi architectonici e decorativi, Bukarest.

Boschung, D. (1993a), Die Bildnisse des Augustus, Berlin. 
Boschung, D. (1993b), “Die Bildungstypen der julisch-claudischen Kaiserfamilie: ein kritischer Forschungsbericht", JRA 6: 39-79

Boschung, D. (2002), Gens Augusta. Untersuchungen zu Aufstellung, Wirkung und Bedeutung der Statuengruppen des julisch-claudischen Kaiserbauses, Mainz.

Boucher, S. (1987), "Limage et les fonctions du dieu Sucellus", Caesarodunum 23: 77-85.

Boulanger, A. (1923), Aelius Aristide et la sophistique dans la province d'Asie au II siècle de notre ère, Paris.

Bowersock, G.W. (1969), Greek Sophists in the Roman Empire, Oxford.

Braancamp Freire A. (ed.) (1916), Notícias da Vida de André de Resende pelo Beneficiado Francisco Leitão Ferreira, Lisboa.

Bracco, V. (1985), "Il tabellarius di Polla", Epigraphica 47: 93-97.

Brandão, M. (1937), Documentos de D. João III, I, Coimbra.

Brandt, H. (1998), Geschichte der römischer Kaiserzeit. Von Diokletian und Konstantin bis zum Ende der konstantinische Dynastie (264-363), Berlin.

Briant, P. (2002), From Cyrus to Alexander. A History of the Persian Empire, Winona Lake.

Brown, B.R. (1957), Ptolemaic Paintings and Mosaics and the Alexandrian style, Cambridge.

Brown, T. S. (1946), "Euhemerus and the Historians", HThR 39: 259-274.

Brun, J.-P. (1986), L'oléiculture antique en Provence. Les huiliers du departement du Var (RANArb suppl. 15), Paris.

Brun, J.-P. (2003), Le vin et l'huile dans la Méditerranée antique. Viticulture, oléiculture et procédés de transformation, Paris.

Brun, J.-P. (2004), Archéologie du vin et de l'huile dans l'empire romain, Paris.

Brun, J.-P. (2005), Archéologie du vin et d'buile en Gaule romaine, Paris.

Bruneau, P. (1985), "Deliaca. Iconographie. L'image de Delos personifiée e pyxides de Spina”, BCH 109: 551-556.

Búa, C. (1997), "Dialectos indoeuropeos na franxa ocidental hispânica”, in G. Pereira (ed.), Galicia fai dous mil anos. O feito diferencial galego, volumen I. Historia, Santiago de Compostela, 51-99.

Buck, R. J. (1979), A History of Boeotia, Edmonton.

Bulloch, A. W (1985), Callimachus. The Fifth Hymn, Cambridge.

Bulloch, A. W (2010), "Hymns and Encomia", in J. J Clauss and M. Cuypers (eds.), A Companion to Hellenistic Literature, Malden/Oxford, 166-180.

Burazacchini, G. (ed.) (2005), Troia tra realtà e legenda, Parma.

Burckhardt J. (1949), The Age of Constantine the Great, Berkeley. 
Burke, P. (1987 3a ed.), The italian Renaissance culture and society in Italy, Cambridge.

Burkert, W. (1991), Mito e Mitologia, Ed. 70, Lisboa.

Burkhalter-Arce, F. (2002), "Le tarif de Coptos". La douane de Coptos, les fermiers de l'apostolion et le préfet du desert de Bérénice”, Topoi Supp. 3: 199-233.

Burnett, A. P. (2005), Pindar's Songs for Young Athletes of Aigina, Oxford.

Bury, J. B., Cook, S. A., Adcock ,F. E. (eds.), The Cambridge Ancient History, Vol. 4, Cambridge.

Butcher, K. (2003), Roman Syria and the Near East, London.

Buxton, R. (ed.) (1999), From Myth to Reason? Studies in the Development of Greek Thought, Oxford.

Caccamo Caltabiano, M. (2003), "Messana/Tyche sulle monete della città dello stretto", in Archeologia del Mediterraneo. Studi in onore di Ernesto De Miro, Roma, 139-149.

Cadotte, A. (2007), La romanisation des dieux. L'interpretatio romana en Afrique du Nord sous le Haut-Empire (Religions in the Graeco-Roman world 158), Leiden.

Caiado, H. (1745), Eclogae et Sylvae et Epigrammata, in Pe. A. dos Reis, Corpus illustrium poetarum Lusitanorum, qui latine scripserunt, Lisboa.

Cairns, D. L. (2010), Bacchylides: five epinician odes (3, 5, 9, 11, 13), Cambridge.

Camia, F. (2011), Theoi sebastoi. Il culto degli imperatori romani in Grecia (provincia Achaia) nel secondo secolo D.C., Athinai.

Caldera de Castro, M. D. P. (1978), "Una sepultura de cupa hallada en Mérida. (Consideraciones acerca de estos monumentos funerarios)", Habis 9: 455-463.

Calderón Dorda, E., De Lazzer, A., Pellizer, E., (eds.) (2003), Corpus Plutarchi Moralium, Naples.

Calvino, I. (1996), "Diario americano, 1959-1966”, in Eremita a Parigi. Pagine autobiografiche, Milano, 20-124.

Calvino, I. (1996a), Città invisibili, Milano.

Camargos, M. (2001), Villa Kyrial: crônica da Belle Époque paulistana, São Paulo.

Cameron A. (1993), The later Roman empire: AD 284-430, Cambridge.

Cantemir, D. (2006), The Salvation of the Wise Man and the Ruin of the Sinful World [...], ed., trans., notes, indices Ioana Feodorov, Editura Academiei, Bucuresti.

Cantemirius, D. (1973), Descriptio antiqui et hodierni status Moldaviae/ Dimitrie Cantemir, Descrierea Moldovei, trans. Gh. Gutu, introd. Maria Holban, hist. com. N. Stoicescu, cartographical study Vintilă Mihailescu, index Ioana Constantinescu, note D. M. Pippidi, Bucuresti. 
Bibliografia

Cantemirius, D. (2006), Descriptio antiqui et hodierni status Moldaviae/ Dimitrie Cantemir, Principele Moldovei, Descrierea stării de odinioară şi de astăzi a Moldovei, ed., trans. Dan Slusanschi, Bucuresti.

Cantineau, J. (1935), Grammaire du palmyrénien épigraphique, Le Caire.

Carcopino, J.(s/d), A vida quotidiana em Roma no apogeu do Império (trad A. J. Saraiva), Lisboa.

Cardim Ribeiro, J. (2002), "Soli Aeterno Lunae. O santuário", Religiões da Lusitânia. Loquuntur Saxa, Lisboa, 235-239.

Cardim Ribeiro, J. (2005), "O deus sanctus Endovellicus durante a romanidade. Uma interpretatio local de Faunus-Silvanus?”, Paleohispanica 5: 721-766.

Carlier p. (1990), Démosthène, Paris.

Carneiro, A., d'Encarnação, J., de Oliveira, J., Teixeira, Cl. (2008), "Uma iscrição votiva em lengua lusitana”, Palaeobispanica 8: 167-178.

Caro, A. (2009), “Una fase decisiva en la evolución de la publicidad: la transición del producto a la marca", Pensar la publicidad, III, 2: 109-114.

Caro, A. (2010), Comprender la publicidad, Barcelona.

Cartledge, P. (2009), Ancient Greek Political Thought in Practice, Cambridge.

Carvalho, J. de (1947-1948), Estudos sobre a cultura portuguesa do século XVI, 2 vols. Coimbra.

Carvalho, J. de (1949), Estudos sobre a cultura portuguesa do século XV, Coimbra.

Cascudo, L. C. (1974), Prelúdio e fuga do real, Natal.

Cascudo, L. C. (1983), Civilização e Cultura, pesquisas e notas de etnografia geral, Belo Horizonte.

Cascudo, L. C. (1983, 2ª ed.), Anúbis e outros ensaios. Mitologia e folclore, Rio de Janeiro, Natal.

Cascudo, L. C. (1987), História dos nossos gestos, Belo Horizonte, São Paulo.

Cascudo, L.C. (1966), "História de um livro perdido", Arquivos do Instituto de Antropologia "Câmara Cascudo" 2.1-2: 5-19.

Castelli, E. (1951) (ed.), Umanesimo e Scienza politica. Atti del congresso Internazionale di Studi Umanistici, Roma-Firenze, 1949, Milano.

Castillo, C. (1998), "Los flamines provinciales de la Bética", REA 100: 437-460

Cawkwell, G. (2005), The Greek Wars. The Failure of Persia, Oxford.

Cesarano, M. (2015), In honorem domus divinae. Introduzione allo studio dei cicli statuari giulio-claudii a Roma e in Occidente, Roma.

Clauss, M. (1979), Kaiser und Gott: Herrscherkult im romischen Reich, Berlin.

Chamie, M. (2009), Paulicéia dilacerada, Ribeirão Preto.

Chaniotis, A. (2009), "The Dynamics of Rituals in the Roman Empire", in O. 
Hekster, S. Schmidt-Hofner, Chr. Witschel (eds.), Ritual Dynamics and Religious Change in the Roman Empire. Proceedings of the Eight Workshop of International Network Impact of Empire, Leiden, Boston, 3-29

Charles-Picard, G., Rougé, J. (1969), Textes et documents relatifs à la vie economique et sociale dans l'Empire romain, Paris.

Chassaing, M. (1961), "Les barillets frontiniens“, RAE 12: 7-33, 89-106.

Chelotti, M. (2003), Regio II, Apulia et Calabria, Venusia (Supplementa Italica 20), Roma.

Cherry, D. (1998), Frontier and Society in Roman North Africa, Oxford.

Chevallier, R. (1972), Les voies romaines, Paris.

Chevallier, R. (1988), Voyages et déplacements dans l'Empire romain, Paris.

Chiarelli, G. (1932), 'Il 'De regno' di Francesco Patrizi', Rivista internazionale di filsosofia del diritto, Anno XII. (Nov-Dec.): 716-738.

Cistercienses (Os). Documentos primitivos. Texto latino e tradução brasileira. (1997) Introdução e bibliografia Irmão François de Place, Tradução de Irineu Guimarães, Musa, S. Paulo; Lúmen Christi, Rio de Janeiro 1997.

Clauss, J., Cuypers, M. (eds.) (2010), A Companion to Hellenistic Literature, Chichester, West Sussex.

Clavel-Lévêque, M. et alii (eds.) (1993), Siculus Flaccus. Les conditions des terres, Nápoles.

Clavel-Lévêque, M. et alii (eds.) (1996), Hygin l'arpenteur. L' établissement des limites, Nápoles.

Clayton, P.A. (1989), Le sette Meraviglie del mondo, Torino. (The Seven Wonders of the Ancient World, London, 1988).

Cogitore, I. (1996), “Séries de dédicaces italiennes à la dynastie julio-claudienne”, MEFRA 104 : 817-870.

Colasso, F. (1951), "Umanesimo giuridico", in E. Castelli (ed.), Umanesimo e Scienza politica (Atti dei Congresso Internazionale di Studi Umanistici, Roma-Firenze, 1949), Milano, 57-58.

Colecchia, A., Bertolani, G. B., Marcante, A. et alii (2004), L'Alto Garda occidentale dalla preistoria al postmedioevo. Archeologia, storia del popolamento e trasformazione del paesaggio (Documenti di archeologia, 36), Mantova.

Colonna, G. (1980), "Virgilio, Cortona e la leggenda etrusca di Dardano", Archeologia Classica 32: 1-15.

Conger, G. P. (1952), "Did India influence Early Greek Philosophies?", Philosophy East and West 2.2: 102-128.

Conti, S. (1997), "Dinastia giulio-claudia a Roselle: una serie di dediche imperiali in Etruria”, Ann. Fac. Lett. e Filos. Univ. Siena 18: 101-127. 
Conti, S. (1998), Rusellae, Suppl. It. n. s. 16, Roma.

Cook, J. M. (1971), Os Gregos na Iónia e no Oriente, Lisboa.

Cooley, A. E. (ed.) (2002), Becoming Roman, Writing Latin? Literacy and Epigraphy in the Roman West. JRA Suppl. Ser. 48, Portsmouth.

Cooley, A. E. (2002), "The survival of Oscan in Roman Pompeii”, in E. A. Cooley (ed.), Becoming Roman, Writing Latin? : Literacy and Epigraphy in the Roman West, JRA Suppl. Ser. 48: 77-86.

Cordovana, O. D., Galli, M. (eds.) (2007), Arte e memoria culturale nell'età della Seconda Sofistica, Catania.

Corell, J. (1989), “Notas sobre epigrafía romana del País Valenciano”, APL 19: 271-281.

Costa, A. D. S. (1969), Estudantes portugueses na reitoria do Colégio de S. Clemente de Bolonha na primeira metade do século XV, Lisboa.

Costa, A. D. S. (1990), Portugueses no Colégio de S. Clemente e Universidade de Bolonha durante o século $X V$, vol. I, Bolonia.

Coulanges, F. de. (1971, 10ª ed.), A cidade antiga, Trad. e glossário de Fernando de Aguiar, Livraria Clássica Editora, Lisboa.

Crawford, M. H., Reynolds, J. M. (1979), “The Aezani copy of the Prices Edict”, Zeitschrift für Papyrologie und Epigraphik 34: 163-210.

Crystal, D. (2000), Language death, Cambridge.

Curado, F. P. (1985), "Inscrição rupestre de Freixo de Numão”, Ficheiro Epigráfico 11: no48.

David, B., Thomas J. (eds.) (2008), Handbook of landscape archaeology (World archaeological congress research handbooks in archaeology, 1), Walnut Creek.

Davie, J. N. (1982), "Theseus the king in fifth-century Athens", GE'R 29.1: 2534.

DCPH = M. P. García-Bellido y C. Blázquez (2001), Diccionario de cecas y pueblos hispánicos, Madrid.

De Bernardo Stempel, P. (2008), "More names, fewer deities. Complex theonymic formulas and the three types of interpretation", in Divindades indígenas em análise. Divinités pré-romaines. Bilan et perspectives d'une recherche. Actas do VII workshop FERCAN, Cascais, 25-27.5.2006, Coimbra, 65-73.

De Hoz, J. (2001), "La lengua de los íberos y los documentos epigráficos en la comarca de Requena-Utiel”, in A. J. Lorrio (ed.), Los íberos en la comarca de Requena-Utiel (Valencia), Madrid, 49-62.

De Hoz, J. (2010), Historia lingüistica de la Península Ibérica en la Antigüedad. I. Preliminares y mundo meridional prerromano, Madrid. 
De Hoz, M. P. (1997), “Epigrafía griega en Hispania”, Epigraphica 59: 29-93.

De Labriolle, P. (1934), La reaction païenne, Paris.

De Martino, D. (2010), "Spot, etica e letteratura", La nuova ricerca. Pubblicazione annuale del Dipartimento di Linguistica, Letteratura e Filologia moderna dell'Università degli studi di Bari, anno XIX. 19, 117-128.

De Martino, D. (2010 bis), "Automobili da mito", in F. De Martino (ed.), Antichità Ẽ pubblicità, Bari, 443-522.

De Martino, D. (2011), Io sono Giulietta. Letterature E' miti nella pubblicità di auto, Bari.

De Martino, D. (2012), "Una forma de subversión del mito literario: de la novela a la publicidad", in J. M. Losada Goya, M. Guirao Ochoa (eds.), Myth and Subversion in the Contemporary Novel, Cambridge, 421-436.

De Martino, D. (2013), Dante E̋ la pubblicità, Bari.

De Martino, F., Vox, O. (1996) (eds.), Lirica greca, vol. 3, Bari.

De Ruyt, Cl. (1983), Macellum. Marché alimentaire des romains, Louvain-laNeuve.

De Santerre, H. H. (1976), "Athènes, Délos et Delphes d'après une peinture de vase à figure rouges du V siècle avant J.-C.”, BCH 100: 291-298.

De Vos, M., Andreoli, M., Attoui, R. et alii (2007), "Cilicia campestris orientale. Leconomia rurale e la trasformazione del paesaggio intorno al Karasis", in Geografia e viaggi nell'antichità. Atti del convegno internazionale di studi (Certosa di Pontognano, 9-10 ottobre 2005), Siena, 13-39.

Degl'Innocenti Pierini, R. (2012), "Le città personificate nella Roma repubblicana: fenomenologia di un motivo letterario tra retorica e poesia", in G. Moretti, A. Bonandini (eds.), Persona ficta. La personificazione allegorica nella cultura antica, fra letteratura, retorica e iconografia, Trento, 215-247.

Desbat, A. (1991), "Un bouchon de bois du Ier s. aprés J.-C. recueilli dans la Saône à Lyon et la question du tonneau à 1'époque romaine", Gallia 48: 319-336.

Dias, P. B. (2011 2a ed.), “Notas introdutórias”, in J. G Freire, A versãolatina por Pascásio de Dume dos Apophtegmata Patrum, Coimbra, 1-34.

Dias, P. B. (2012), “Cristianismo e responsabilidade cristã na queda de Roma”, in F. Oliveira et alli (coords.), A queda de Roma e o alvorecer da Europa, Coimbra, 43-67.

Dias P. B. (2013), "O legado de Constantino na identidade da Europa cristã: dois casos de estudo", in M. C. Pimentel e P. Farmhouse Alberto (orgs.), Vir bonus peritissimus aeque. Estudos de homenagem a Arnaldo do Espirito Santo, Lisboa, 455-463 
Díaz, B. (2008), Epigrafía latina republicana de Hispania, Barcelona.

Dittenberger, W., Purgold, K. (1896), Inschriften von Olympia, Berlin.

Dixon, R. M. W. (1997), The rise and fall of languages, Cambridge.

Domăneanțu, C. (1993), "Un sanctuaire hellénistique du site de Nuntaşi II (comm. d'Istria, dep. De Constanța)”, Dacia 37: 59-78.

Dörfler, W., Evans, A., Löhr, H. (1998), “Trier, Walramsneustrasse. Untersuchungen zum römerzeitlichen Landschaftswandel im Hunsrück-Eifel-Raum an einem Beispiel aus der Trierer Talweite“, in Studien zur Archäologie der Kelten, Römer und Germanen in Mittel- und Westeuropa. Alfred Haffner zum 60. Geburtstag gewidmet, Rahden, 119152.

Dubuisson, M. (1981), "Utraque lengua”, L'Antiquité Classique 50: 274-286.

Dubuisson, M. (1982), "Y a-t-il une politique linguistique romaine?”, Ktéma 7: 197-210.

Duchesne, L. (1887), "Le concile d'Elvire et les flamines chrétiens", Mélanges Renier, Paris, 159-174.

Dunkle, J. R. (1969), “The Aegeus episode and the theme of Euripides' Medea", TAPhA 100: 97-107.

Durán Fuentes, M. (2005), La construcción de puentes romanos en Hispania, Santiago de Compostela.

Eck, W. (2006), "Herrschaft und Kommunikation in antiken Gesellschaften. Das Beispiel Rom", in U. Peter, S. J. Seidlmayer (eds.), Mediengesellschaft Antike? Information und Kommunikation vom Alten Ägypten bis Byzanz, Berlin, 11-33.

Eco, U. (2013), Storia delle terre e dei luoghi leggendari, Milano.

Eddy, S.K. (1968), “Four Hundred Sixty Talents Once More”, CP 63: 184-95.

Edmonson, J. (1997), “Two dedications to Divus Augustus and Diva Augusta from Augusta Emerita and the early development of the imperial cult in Lusitania", MM 38: 89-105.

Edmondson, J. (2002), "Writing latin in the province of Lusitania", in A. E. Cooley (ed.), Becoming Roman, Writing Latin? Literary and Epigraphy in the Roman West, JRA Suppl. Ser. 48: 41-60.

Ehrenberg, V. (1973, 2a ed.), From Solon to Sócrates, Londres.

Ehrenberg, V. (1976), L'État grec, Paris.

Ehrhardt, N. (1988), Milet und seine Koloniei. Vergleichende Untersuchung der kultischen und politischen Einrichtungen, ed. a II-a, Frankfurt, Main-Bern, New York, Paris.

Elliger, W. (1975), Die Darstellung der Landschaft in der griechischen Dichtung, Berlin, New York. 
Elliott, Th. (1990), “The Language of Constantinian Propaganda”, TAPhA 120: 349-353.

Encarnação, J. d' (1984), Inscrições romanas do Conventus Pacensis, Coimbra.

Erasmo, D. (1703), Opera omnia (in decem tomos distincta), Recognovit Joannes Clericus, Leiden.

Erodoto (1988), Le Storie. Libro I. La Lidia ela Persia. Acura di David Astheri, Milano.

Erskine, A. (ed.) (2003), A Companion to the Hellenistic World, Oxford.

Espérandieu, E. (1907-1981), Recueil général des bas-reliefs, statues et bustes de la Gaule romaine, Paris.

Estarán, M. J. (2012), “Las estampillas ibérico-latinas K.5.4”, Palaeohispanica 12: $73-90$

ET = Rix, H. (1991), Etruskische Texte, Tübingen.

Étienne, R. (1958), Le culte impérial dans la Péninsule ibérique d’Auguste à Diocletien, Paris.

Étienne, R. (1973), "Les syncrétismes dans la Péninsule Ibérique à l'époque impériale", in Les syncrétismes dans les religions grecque et romaine, Paris, 153-163.

Étienne, R., Fabre, G.; Lévêque, P. et M. (1976), Fouilles de Conimbriga, vol. II, Épigraphie et Sculpture, Paris.

Étienne, R., Fabre, G., Le Roux, P., Tranoy, A. (1976), "Les dimensions sociales de la romanisation dans la Péninsule Ibérique des origines à la fin de l'Empire", in D. M. Pippidi (ed.), Assimilation et résistance à la culture gréco-romaine dans le monde ancien. Travaux du VI Congrès International d'Études Classiques, Bucureşti, Paris, 95-107.

Étienne, R., Mayet, F. (2000), Le vin hispanique, Paris.

Evans, J. A. S. (1981), "Notes on the debate of the Persian Grandees in Herodotus 3, 80-82”, QUCC 36: 79-84.

Evers, C. (1994), Les portraits d'Hadrien. Typologie et ateliers, Bruxelles.

Ewald, C., Norena, C. F. (eds.) (2010), The Emperor and Rome: Space, Representation, Ritual, Cambridge.

Fabre, G., Mayer, M., Rodà, I. (1991), Inscriptions romaines de Catalogne, III, Paris.

Fayer, C. (1976), Il culto della dea Roma. Origine e diffusione nell'Impero, Pescara.

Fearn, D. (2007), Bacchylides. Politics, performance, poetic tradition, Oxford.

Fernandes, L., Carvalho, P., Figueira, N. (2009), "Divindades indígenas numa ara inédita de Viseu", Palaeohispanica 9: 143-155.

Fernández Gallardo, L. (2002), Alonso de Cartagena. Una biografía politica en la Castilla del siglo XV, Valladolid. 
Fernández Gallardo, L. (2008), “Alonso de Cartagena y el Humanismo”, La Corónica 37.1: 175- 215.

Ferraz, C. (2002), "Conjunto de oito aras provenientes do Lararium de Centum Celas", in V. L. Raposo, J. R. Ferreira (Coords.), Religióes da Lusitânia. Loquuntur Saxa, Lisboa, 467-469.

Ferreira, J. R. (1988), "Grécia e Roma na Revolução Francesa”, Revista de História das Ideias 10: 203-234.

Ferreira, J. R. (1990), A democracia na Grécia Antiga, Coimbra.

Ferreira, J. R. (1990a), Participação e poder na democracia grega, Coimbra.

Ferreira, J. R. (1993), Hélade e Helenos I - Génese e Evolução de um Conceito, Coimbra.

Ferreira, J. R. (2004 2a ed.), A Grécia Antiga. Sociedade e Politica, Lisboa.

Ferreira, J. R., (1991), "Presença da Grécia e de Roma na Revolução Francesa", in Actas do colóquio A Recepção da Revolução Francesa em Portugal e no Brasil, Porto, vol. I, 75-96.

Ferri, S. (1976), "Luci e ombre sulla interpretatio romana", in Convegno internazionale "Renania romana" Roma 14-16 aprile 1975, Roma, 125133.

Ferrill, A. (1978), "Herodotus on tyranny", Historia 27.3: 385-398.

Figueira, T. J. (1998), The Power of Money: Coinage and Politics in the Athenian Empire, Philadelphia.

Figueira, T. J. (2003), "Economic Integration and Monetary Consolidation in the Athenian Arkhê", in G. Urso (ed.), Moneta, Mercanti, Banchieri. I precedenti greci e romani dell'Euro, Pisa, 71-92.

Figueira, T. J. (2005), "The Imperial Commercial Tax and the Finances of the Athenian Hegemony”, Incidenza dell'antico 3: 83-133.

Figueira, T. J. (2006), "Reconsidering the Athenian Coinage Decree", AIIN 52: 9-44.

Figueira, T. J. (2011), "The Athenian Naukraroi and Archaic Naval Warfare”, Cadmo. Revista de História Antiga 21: 183-210.

Figueira, T. J. (forthcoming[a]), "Archaic Naval Warfare”, in N. Birgalias (ed.), Great is the Power of the Sea: The Power of Sea and Sea Powers in the Greek world of the Archaic and Classical Periods, Athens.

Figueira, T. J. (forthcoming[b]), "The Aristeidian Tribute on the Peace of Nikias", in S. Jensen, T. Figueira (eds.), Athenian Hegemonic Finances, Swansea.

Figueira, T. J. (forthcoming[c]), "Community Wealth and Military Might in Periclean Athens", in A.L. Pierris (ed.), Mind, Might, Money: The Secular Triad in Classical Athens, Patras. 
Figueira, T. J. (forthcoming[d]), “Aigina: Island as Paradigm”, in A. Powell and K. Meidani (eds.), The Eyesore of Aigina: Anti-Athenian Attitudes in Greek, Hellenistic and Roman History, Swansea.

Figueiredo, R., Lamounier, B. (1996), As cidades que dão certo, Brasília.

Finley, M.I. (1966), The Ancient Greeks. An introduction to their life and thought, Londres. Trad. port.: Os Gregos Antigos (Lisboa, 2a ed. 1988).

Finley, M. I. (1973, 2a ed.), Democracy, ancient and modern, London.

Finley, M.I. (1973a), The ancient economy, London.

Finley, M.I. (1982), Authority and legitimacy in the classical city-state, Kobenhavn.

Fishwick, D. (1970), "Flamen Augustorum”, HSCPh 74: 299-312.

Fishwick, D. (1982), "The altar of Augustus and the municipal cult of Tarraco", MM 23: 222-233

Fishwick, D. (2002), The Imperial Cult in the Latin West, 3/2, Provincial Cult I The Provincial Priesthood, Leiden.

Fishwick, D. (2005), The Imperial Cult in the Latin West. Studies in the Ruler Cult of the Western Provinces of the Roman Empire, Leiden, Boston.

Fitton, J. W. (1961), "The Suppliant Women and the Herakleidai of Euripides", Hermes 89.4: 430-461.

Flower, M. F. (2007), "Appendix R: The Size of Xerxes Expeditionary Force," in Robert B. Strassler (ed.), The Landmark Herodotus: The Histories, New York, 819-23.

Fonseca, L. A. (1982), O Condestável D. Pedro de Portugal, Porto.

Fontanella, F. (2008), "The Encomium on Rome as a response to Polybius' doubts about the Roman Empire", Columbia Studies in the Classical Tradition 33: 203-216.

Forni, G. (1973), "El culto de Augusto en el compromiso oficial y en el sentimiento oriental", BSAA 39: 105-113.

Forni, G. (1994), Scritti vari di Storia, Epigraphia e antichità romane, Roma.

Franck, A. D. (1864), Réformateurs et publicistes de l'Europe: Moyen ÂgeRenaissance, Paris.

French, A. (1972), "The Tribute of the Allies", Historia 21: 3-20.

Fuentes, M. J. (1986), Corpus de las inscripciones fenicias, púnicas y neopúnicas de Hispania, Barcelona.

Gabba, S., Drioton, É. (1954), Peintures à fresques et scènes peintes a Ermoupolis Ouvest (Touna el-Gevel), Le Caire.

Gaffiot, F. (s/d), Dictionnaire latin-français, Paris.

Gagé, J. (1936), "Le templum Urbis et les origines de l'idée de Renovatio", in Mélanges Franz Cumont, Bruxelles, 151-187. 
Gagé, J. (1955), Apollon romain. Éssai sur le culte d'Apollon et le développement du "ritus Graecus" à Rome des originrs à Auguste, Paris.

Gagé, J. (1968), "Basiléia”. Les Césars, les rois d'Orient et les "mages", Paris.

Gagé, J. (1974), "Le solemne Urbis du 21 avril au III" siècle ap. J.-C.: Rites positives et speculations séculaires", Mélanges d'histoire de religions offerts à Henri-Charles Puech, Paris, 225-241.

García Bellido, M. P. (1993), "Sobre el culto de Volcanus y Sucellus en Hispania. Testimonios numismáticos", in F. Burkhater, J. Arce (eds.), Bronces y religión romana. Actas del XI Congreso internacional de bronces antiguos, Madrid mayo - junio 1990, Madrid, 161-170.

García Iglésias, L. (1976), "Autenticidad de la inscripción de municipios que sufragaron el puente de Alcántara”, Revista de Estudios Extremeños 32.2: 263-276.

García Jurado, F. (2007), Aulo Gelio, Noches Aticas. Antología, Madrid.

García Romero, F. (2002), "Pervivencia de Penélope”, in C. Morenilla Talens, F. De Martino (eds.), El perfil de les ombres, Bari, 187-204.

García Soler, M. J. (2010), "Gastronomia e pubblicità nella Grecia antica”, in F. De Martino (ed.), Antichità Ẻ pubblicità, Bari, 345-366.

Garin, E. (1955), "Ricerche sulle traduzioni di Platone nella prima metà del XV secolo", Medioevo e Rinascimento, Studi in onore di B. Nardi, Firenze.

Garin, E. (1966), Storia della filosofia italiana, Torino.

Garriguet, J. A. (2004), "Grupos estatuarios imperiales de la Bética: la evidencia escultórica y epigráfica”, in Actas de la IV reunión sobre escultura romana en Hispania, Madrid, 67-101.

Gasperini, L. (1977), “L’Augusteo di Firmo Piceno in un'epigrafe da rileggere”, AFML 10: 57-87.

Gasperini, L. (2008), "L’Augusteo di Forum Clodii”, en L. Gasperini, G. Paci, (eds.), Nuove ricerche sul culto imperiale in Italia, Tivoli, 91-134.

Gasperini, L., Paci, G. (eds.) (2008), Nuove ricerche sul culto imperiale in Italia, Tivoli.

Gaudemet J. (1947), “La législation religieuse de Constantin”, Rérue d' Histoire de l'Église de France 122: 25-61.

Genette, G. (1997), Palinsesti. La letteratura di secondo grado, Torino.

Gentili, B. (ed.) (1995), Pindaro. Le pitiche, Milano.

Ghedini, F. (2000), "Filostrato Maggiore come fonte per la conoscenza della pittura antica”, Ostraka 9.1: 75-197.

Giachero, M. (ed.) (1974), Edictum Diocletiani et Collegarum de pretiis rerum venalium in integrum restitutum e latinis gracisque fragmentis, 1-2, Génova. 
Gico, V. (1998), "Luís da Câmara Cascudo: perfil bibliográfico", in L. C. Cascudo, Ontem. (Maginaçôes e notas de um professor de provincia), Natal.

Gigli, D. (1985), Metafora e poetica in Nonno di Panopoli, Firenze.

Gilles, K. J. (1987), "Römische Glasgefäße", in AA.VV., 2000 Jahre Weinkultur an Mosel-Saar-Ruwer. Denkmäler und Zeugnisse zur Geschichte von Weinbau, Weinhandel, Weingenuß, Trier, 143-145.

Gilles, K. J. (1987b), “Trierer Weinkeramik”, in AA.VV., 2000 Jabre Weinkultur an Mosel-Saar-Ruwer. Denkmäler und Zeugnisse zur Geschichte von Weinbau, Weinhandel, Weingenuß, Trier, 132-133.

Gilles, K. J., König, M., Schumann, F. (1995), Neuere Forschungen zum römischen Weinbau an Mosel und Rhein (Schriftenreihe des Rheinischen Landesmuseums Trier, 11), Trier.

Gilson, É. (1983, 6ª ed.), Le thomisme, Paris.

Gómara, M. (2007), "Una inscripción paleohispánica sobre cerámica altoimperial en Cascante (Navarra)", Palaeohispanica 7: 263-268.

Gomes, S.A. (1998), Visitações a mosteiros cistercienses em Portugal. Séculos XVe XVI, Ministério da Cultura - IPPAR, Lisboa.

Gomes, S. A. (2000), "Revisitação a um velho tema: a fundação do Mosteiro de Alcobaça”, in Cister: Espaços Território e Paisagens. Colóquio Internacional, 16-20 Junho de 1998, Mosteiro de Alcobaça. Actas. I, Lisboa, 27-72.

Gomes, S. A. (2000), O mosteiro de Alcobaça na transição dos séculos XIV e XV: o protagonismo de D. João Dornelas, in Cister. Espaços, Territórios, Paisagens. Colóquio Internacional. 16-20 Junho 1998. Mosteiro de Alcobaça, Lisboa, 73-88.

Gomes, S. A. (2006), D. Afonso V, Círculo de Leitores-Colecção Reis de Portugal, Lisboa.

Gómez García, C. (2010), "La configuración de la ciudad de Berlin”, in J. M. Losada Goya (ed.), Mito y mundo contemporáneo. La recepción de los mitos antiguos, medievales y modernos en la literatura contemporánea, Bari , 617626.

González Rolán, T., P. Saquero Suárez-Somonte, P. (2001), “E1 Humanismo italiano en la Castilla del cuatrocientos: estudio y edición de la versión castellana y del original latino del De infelicitate principum de Poggio Bracciolini “, Cuadernos de Filología Clásica. Estudios Latinos 21: 115-150.

González Rolán, T., Moreno Hernández, A., Saquero Suárez-Somonte, P. (2000), Humanismo y teoría de la traducción en España e Italia en la primera mitad del siglo XV. Edición y estudio de la Controversia Alphonsiana (Alfonso de Cartagena vs. L. Bruni y P. Candido Decembrio), Madrid.

Gorrochategui, J. (1987), "Situación lingüística de Navarra y aledaños en la antigüedad a partir de las fuentes epigráficas", Primer Congreso General de Historia de Navarra II, Pamplona, 435-445. 
Gorrochategui, J. (2014), "Nueva inscripción funeraria celtibérica procedente de Clunia”, Palaeobispanica 14: 229-236.

Gorrochategui, J.y Vallejo, J. M. (2010), “Lengua y onomástica. Las inscripciones lusitanas", Iberografías 6: 71-80.

Gose, E. (1976), Gefäßtypen der römischen Keramik im Rheinland, Köln.

Graham, A.J. (1964), Colony and Mother City, Manchester.

Grenier, A. (1934), Manuel d'archéologie gallo-romaine 2, Les routes, Paris.

Gros, P., Marin, M., Zink, M. (eds.) (2015), Auguste, son époque et l'Augusteum de Narona. Actes du colloque organisé à l 'Académie des Inscriptions et Belles-letres le 12 décembre 2014, Paris.

Gualandi, M. L. (2001), Le fonti per la storia dell'arte - I. L'antichità classica, Roma.

Guarducci, M. (1974), Epigrafia greca, vol. III, Roma.

Guarducci, M. (1978), Epigrafia greca, vol. IV, Roma.

Guerra, A., Schatner, T. (2010), "El foro y el templo de Lancia Oppidana: nueva interpretación de Centum Celas (Belmonte)", in T. Mogale Basarrate (ed.) Ciudad y Foro en Lusitania Romana, Mérida, 333-342.

Guilaine, J. (cur.) (1991), Pour une archéologie agraire: à la croisée des sciencies de l'homme et de la nature, Paris.

Guilmartin, J. F. (2002), Galleons and Galleys, London.

Guilmartin, J. F. (2003), Gunpowder and Galleys. Changing Technology and Mediterranean Warfare at Sea in the Sixteenth Century, 2nd ed., Annapolis.

Hall, J. M. (1997), Ethnic identity in Greek Antiquity, Cambridge.

Hanell, K. (1934), Megarische Studien, Lund.

Hänlein-Schäfer, H. (1985), Veneratio Augusti. Eine Studie zu den Tempeln der ersten römischen Kaisers, München.

Hanley, R. (2000), Villages in Roman Britain, Princes Risborough.

Hansen, H. M. (1991), The Athenian Democracy in the age of Demosthenes. Structure, Principles and Ideology, Oxford.

Hardy, E. G. (1925), "The Lex Mamilia Roscia Peducaea Alliena Fabia”, The CQ 19 (3/4): 185-191.

Harris, E. (1995), Aeschines and Athenian Politics, Oxford.

Harth, H. (1984), Poggio Bracciolini, Lettere, Leo S. Olschki Editore, Florencia.

Hekster, O., Schmidt-Hofner, S., Witschel, Chr. (eds.) (2009), Ritual dynamics and Religious Change in the Roman Empire. Proceedings of the Eighth Workshop of the International Network Impact of Empire, Leiden, Boston.

Helck, W. (1971), Das Bier im alten Ägypten, Berlin. 
Hershowitz, A., (forthcoming), "Patterns in Variation in Tribute Assessment", in S. Jensen, T. Figueira (eds.), Athenian Hegemonic Finances, Classical Press of Wales, Swansea.

Herta, P. (1978), "Bibliographie zum römischer Kaiserkult (1955-1975)", ANRW II 18: 833-910.

Heubeck, A. (ed.) (1983), Omero. Odissea, Volume III (Libri IX-XII), Milano.

Heurgon, J. (1950-1951), "La syntaxe des routiers romains", Bulletin de la Société des Antiquaires de France: 145-154.

Heurgon, M. (1969), "Inscriptions étrusques de Tunisie”, CRAI, 526-551.

Heurgon, M. (1969a), “Les Dardaniens en Afrique”, REL 47: 284-294.

Higbie, C. (2007), "Hellenistic Mythographers", in R. Woodart (ed.), The Cambridge Companion to Greek Mythology, Cambridge, 237-54.

Hignett, C. (1963), Xerxes' Invasion of Greece, Oxford.

Hoffmann, C. (1991), An introduction to bilingualism, London, New York.

Hoffmann, M. (1956), 5000 Jahre Bier, Berlin.

Holban, M., Bulgaru, M. M. A., Cernovodeanu, P. (eds.) (1980-83), Calatori straini despre tarile române (Foreign Travellers about the Romanian Countries), Bucuresti, vol. VII: 1980; vol. VIII: 1983.

Homo, L. (1972), Rome impériale et l'urbanisme dans l'antiquité, Paris.

Hopkinson, N. (1984), “Callimachus' Hymn to Zeus”, CQ 34: 139-148.

Hornblower, S. (2008), A Commentary on Thucydides. Volume I: Books I-III, Oxford - New York.

Houaiss, A. (2001), Dicionário Houaiss da lingua portuguesa, Rio de Janeiro.

Howgego, Chr, Heuchert, V. Burnett, A. (eds.) (2004), Coinage and identity in the Roman provinces, Oxford.

Howgego, Chr. (2004), "Coinage and identity in the Roman provinces", in Chr. Howgego, A. Heuchert y Burnett (eds.), Coinage and identity in the Roman provinces, Oxford, 1-18.

Huizinga, J. (1948), Le déclin du Moyen Âge, Paris.

Hunter, R., Fuhrer, T. (2002), "Imaginary Gods? Poetic Theology in the Hymns of Callimachus", in F. Montanari, L. Lehnus (eds.), Callimaque. Sept Exposés suivis de discussions, Vandoeuvros-Gender, 143-175.

Hurlet, F. (1996), Les collègues du prince au temps d'Auguste et de Tibére: de la légalité républicaine à la légitimité dynastique, Roma.

Hutchinson, G. O. (1988), Hellenistic Poetry, Oxford.

HCT = Gomme, A. J., (1970), A Historical Commentary on Thucydides. vs. 1-2, Oxford. 
Icks, M. (2001), "Priestohood and Imperial Power. The Religious Reforms of Heliogabalus 220-222", in L. de Blois (ed.), Administration, Prosopography and Appointment Policies in the Roman Empire. Proceedings of the First Workshop of the International Network Impact of Empire (Roman Empire, 27 B.C. - A.D. 406), Amsterdam, 169-178.

$I R T=$ Reynolds , J. M., Ward-Perkins, J. B. (1952), Inscriptions of Roman Tripolitania, Rome.

Jackson. K. (1953), Language and history in Early Britain, Edinburgh.

Jacoby, F. (1923), Die Fragmente der griechischen Historiker, Part I-III, Berlin.

Jaeger, W. (s.d), Paideia, Trad. de Artur M. Parreira, São Paulo.

Janko R. (1982), Homer, Hesiod and the Hymns, Cambridge.

Jiménez, A. J. (1995), "La imagen de Teseo en las Suplicantes", in J. A. López Férez (ed.), De Homero a Libanio, Madrid, 145-161.

Johnson, L. (1960), "Natalis urbis and principium anni", TPAPhA 91: 109-120

Julia, D. (1962), "Les monuments funéraires en forme de demi-cylindre dans la province romaine de Tarragonaise", MCVI : 29-54.

Jullian, C. (1926), "Notes gallo-romaines”, Rérue des Études Anciennes 28. 2: 139-151.

Little, K. (2002), "Monasticism and Western Society: from marginality to the establishment and back", Memoirs of the American Academy in Rome 47: 83-94.

Kaimio, J. (1979), The Romans and the Greek Language, Helsinki.

Kalinowski, A. (2007), "A series of honorific statue bases for the Vedii in the market agora at Ephesos (IvE 725, 731, 3076-3078)", in M. Mayer, G. Baratta, A. Guzmán, (eds.), Acta XII Congressus internationalis epigraphiae Graecae et Latinae. Provinciae imperii Romani inscriptionibus descriptae, vol I, Barcelona, 757-762.

Kantiréa, M. (2007), Les dieux et les dieux augustes. Le culte impérial en Grèce sous le Julio-claudiens et les Flaviens, Études épigraphiques et archéologiques, Athènes.

Katz, S. H., Fleming, S. J., McGovern, P. E. (1996), The origins and ancient history of wine. Food and nutrition in history and anthropology 11, Amsterdam.

Kelso W.M. (ed.) (1990), Earth patterns. Essays in landscape archaeology, Charlottesville.

Kerkhecker, A. (1999), Callimachus' Book of "Iambi", Oxford.

Khanoussi, M. (1983), “Nouvelles sépultures d'époque romaine”, in Beschaouch A. et alii (eds.), Recherches archéologiques franco-tunisiennes à Bulla-Regia, I (CEFR 28/I), Roma, 93-106.

Kiss, Z. (1975), L'iconographie des princes julio-claudiens au temps d'Auguste et de Tibère, Varsovie. 
Kleiner, F. S. (1991), "The trophy on the bridge and the Roman triumph over nature", L'Antiquité Classique 60: 182-192.

Koch, J. (2009), Tartessian. Celtic in the South-west at the dawn of history, Aberystwyth.

Koch, J. (2009a), "A case for Tartessian as a Celtic language", Palaeohispanica 9: 339-351.

Kolb, A. (2001), "Tansport and communication in Roman state: the cursus publicus", in C. Adams and R. Laurence (eds.), Travel and Geography in the Roman Empire, Londres - Nova Iorque, 95-105.

Kolb, A. (ed.) (2010), Augustae. Machtbewusste Frauen am römischen Kaiserhof? Herrschaftsstrukturen und Herrschaftspraxis, Berlin.

Kozakai, T. (2000), L'étranger, l'identité. Essai sur l'integration culturelle, Paris.

Kramer, N., Reitz, Chr. (eds.) (2010), Tradition und Erneuerung. Mediale Strategien in der Zeit der Flavier, Berlin, New York.

Kristeller, P. O. (1961, 3a ed.), “The moral thought of Renaissance humanism”, in Chapters in Western civilization, I, New York, 289-335.

Krynen, J. (1981), Idéal du prince et pouvoir royal en France à la fin du Moyen Âge (1380-1440). Étude de la littérature politique du temps, Paris.

Kuhoff, W. (2001), Diokletian und die Epoche der Tetrarchie, Frankfurt.

Künzl, S. (1997), Die Trierer Spruchbecherkeramik. Dekorierte Schwarzfirniskeramik des 3. und 4. Jahrbunderts (Beihefte Trierer Zeitschrift 21), Trier.

Lambert, P. Y. (1994), La langue gauloise, Clamecy.

Lambrino, S. (1937), "La famille d'Apollon à Histria”, Aephem 100: 352-362.

Lambrino, S. (1952), "Les inscriptions de São Miguel de Odrinhas”, Bulletin des Études Portugaises 16: 134-176.

Lasserre, F. (1976), "Hérodote et Protagoras: le débat sur les constitutions", $M H$ 33: 65-84.

Lateiner, D. (1984), "Herodotean historiographical patterning: the constitutional debate", QS 20: 257-284.

Laurence, R. (2001), "Afterword: travel and empire", in C. Adams and R. Laurence (eds.), Travel and Geography in the Roman Empire, Londres / Nova Iorque, 167-176.

Lausberg, H. (1990, 3a ed.), Handbuch der literarischen Rhetorik. Eine Grundlegung der Literaturwissenschaft, Stuttgart.

Lawrance, J. N. H. (1990), "Humanism in the Iberian Peninsula", in A. Goodman, A. Mackay (eds.), The Impact of Humanism on Western Europe, Londres, 220-258.

Lazenby, J. F. (1993), The Defence of Greece, 490-479 B.C., Warminster. 
Leão, D. F. (2012), A Globalização no Mundo Antigo. Do Polites ao Kosmopolites, Coimbra.

Lehmann, K. (1962), "Ignorance and search in the villa of the Mysteries", JRS 52: $62-68$.

Leite de Vasconcelos, J. (1913), Religióes de Lusitania, III, Lisboa 1989.

Leite, S. (ed.) (1963), Estatutos da Universidade de Coimbra (1559), Coimbra.

Lekai, L. J. (1987), Los Cistercienses. Ideales y realidad, Barcelona.

Lemny, S. (2010), Cantemirestii. Aventura europeana a unei familii princiare din secolul al XVIII-lea (Les Cantemir: l'aventure européenne d'une famille princière au XVIIIe siècle, 2006), Iasi, Polirom.

Lesky, A. (1995), História da Literatura Grega, Lisboa.

Leveau, Ph. (1992), "Le territoire agricole d'Arles dans l'antiquité. Relecture de l'histoire économique d'une cité antique à la lumiere d'une histoire du milieu”, in M. Bernardi (cur.), Archeologia del Paesaggio, Firenze, vol. II, 597-636.

Levy, A. M. (2010), Sex Acts in Early Modern Italy: Practice, Performance, Perversion, Punishment, Farnham.

Lewis, D. M, Boardman, J., Hornblower, S., Ostwald, M (eds.) (1994), The Cambridge Ancient History, Volume 6: The Fourth Century BC, Cambridge.

Lewis, D. M. (1994), “The Athenian Tribute Quota Lists, 453-450 BC”, BSA 89: 285-301.

Lima, D. C. (1998, 3a ed.), Câmara Cascudo: um brasileiro feliz. Rio de Janeiro.

Lintott, A. (1992), Judicial reform and land reform in the Roman Republic, Cambridge.

Little K. (2002), "Monasticism and Western Society: from marginality to the establishment and back", Memoirs of the American Academy in Rome 47: 83-94.

Littman, R. J. (1974), The Greek experiment, Imperialism and social conflict 800400 B. C., Londres.

Liverani, P. (1994), "Il ciclo di ritratti del edificio absidato a Roselle", in Roselle: iconografia imperiale e glorificazione Familiare, MDAI, RA 101: 161-163.

Loeschcke, S. (1932), "Römische Denkmäler vom Weinbau an Mosel, Saar und Ruwer", TrZ 7: 42-60.

Loeschcke, S. (1933), Denkmäler vom Weinbau aus der Zeit der Römerherrschaft an Mosel, Saar und Ruwer, Trier.

López Moreda, S. (2009), Aulo Gelio, Noches Aticas, Madrid.

López Vilar, J. (1999-2000), “Consideracions sobre les cupae i altres estructures funeràries afins”, Bullettí Arcqueològic V. 21-22: 65-103. 
Lorenzo Gómez, F. (2010), Un dios entre los hombres. La adoración a los emperadores romanos en Grecia, Barcelona.

Losada Goya, J. M. (ed.) (2010), Mito y Mundo contemporáneo. La recepción de los mitos antiguos, medievales y modernos en la litetatura contemporânea, Bari.

Lucet, B. (1977), Les codifications cisterciennes de 1237 et de 1257, Paris.

Macan, R.W. (1908), Herodotus, The Seventh, Eighth, E Ninth Books, London.

Machado de Assis, J. M. (1971), "Esaú e Jacó", in Machado de Assis, Obra Completa, Rio de Janeiro, José Aguilar Editora.

Maehler, H. (1982), Die Lieder des Bakchylides I (2 vols.), Leiden.

Magioncalda, A. (1991), Lo sviluppo della titolatura imperiale da Augusto a Giustiniano attraverso le testimonianze epigrafiche, Torino.

Magueijo, C. (1970), “A Lex Metallis Dicta”, O Arqueólogo Português série 3, 4: 125-163.

Maltese, V. E.-Cortassa, G. (eds.)(2000), Roma parte del cielo. Confronto tra l'Antica e la Nuova Roma di Manuele Crisolora, Torino.

Mamede, Z. (1970), Luis da Câmara Cascudo: 50 anos de vida intelectual 1918/1968, Natal.

Manconi, D., Catalli, F. (eds.) (2005), Le immagini del potere. Il potere delle immagini. L'uso del ritratto ufficiale nel mondo romano da Cesare ai Severi, Perugia.

Mann, C. (2001), Athlet und Polis im archaischen und frübklassischen Griechenland, Göttingen.

Mantas, V. G. (2008-2009), "A rede viária romana em Portugal. Estado da questão e perspectivas futuras", Anas 21-22: 245-272.

Mantas, V. G. (2011), "Linhas fortificadas e vida quotidiana: da Muralha da China à Muralha do Atlântico", in C. Guardado da Silva (coord.), A Vida quotidiana nas Linhas de Torres Vedras, Torres Vedras, 15-56.

Mantas, V. G. (2012), As vias romanas da Lusitânia, Mérida.

Maquiavel, N. (2010, 8ª ed), O principe, Trad. de Pietro Nassetti, Martin Claret, São Paulo.

Maquiavel, N. (1980), Le Prince de Maquiavel, Traduction et commentaire de C. Roux-Lehman, Paris.

Maravall, J. A. (1972), Estado moderno y mentalidad social (siglos XV a XVII), 2 vols., Madrid.

Marco, F. (1993), "Nemedus Augustus", in I. J. Adiego, J. Siles, J. Velaza, (eds.), Studia Palaeohispanica et Indogermanica J. Untermann ab amicis Hispanicis oblata, Barcelona, 163-178. 
Marco, F. (1996), "Integración, interpretatio y resistencia religiosa en el occidente del imperio", in J. M. Blásquez, J. Alvard (ed.) La romanización en Occidente, Madrid, 217-238.

Marcos Casquero, M. A., Domínguez García, A. (2006), Aulo Gelio, Noches Áticas, vol. I, Universidad de León.

Marcy, G. (1936), Les inscriptions libyques bilingues de l'Afrique du nord, Paris.

Moreno Gallo, I. (2006),Vias romanas: ingenieria y técnica constructiva. Madrid.

Marlière, É. (2001), Le tonneau en Gaule Romaine», Gallia 58: 181-201.

Marlière, É. (2002), L'outre et le tonneau dans l'Occident romain, Montagnac.

Marques, M. A. F. (1998), "A introdução da Ordem de Cister em Portugal”, in Estudos sobre a Ordem de Cister em Portugal, Lisboa.

Marrou, H.-I. (1963), "L'Église dans la première moitié du quatrième siècle”, in L'Église de l'Antiquité tardive 303-604, Paris, 26-35.

Marrou, H.-I. (1965, 6ª ed.), Histoire de l'éducation dans l' Antiquité, Paris.

Marshall, P. K (1983), "Aulus Gellius", in L.D. Reynolds (ed.), Texts and Transmission. A Survey of the Latin Classics, Oxford.

Martínez, A. (1993), "Dos esgrafiados ibéricos sobre una estela romana de Requena (Valencia)", Saguntum 26: 247-251.

Martínez-Pinna, J. (2002), "Los arcadios", in La prebistoria mitica de Roma, Gerión. Anejos 6: 135-167.

Martini, W. (1990), Die archäischen Plastik der Griechen, Darmstadt.

Mastino, A. (1981), Le titolature di Caracalla e Geta attraverso le iscrizioni (indici), Bolonia.

Mattoso, A., (1935 2a ed.), Compêndio de história antiga, Sá da Costa, Lisboa.

Maurice, F. (1930), "The Size of the Army of Xerxes in the Invasion of Greece 480 B.C.", JHS 50: 210-35.

Mayer, M. (1980), "La plasmación lingüística de la pervivencia de los cultos prerromanos en Hispania a través de los formularios epigráficos", Revista Española de Lingüistica 10: 230-231.

Mayer, M. (1993), "El paganismo cívico de los siglos II y III en la Hispania citerior. Su reflejo en la epigrafía", in Ciudad y comunidad civica en Hispania. Siglos II y III d. C. Cité et communauté civique en Hispania, Madrid, 161-175.

Mayer, M. (1995), "El primer horizonte epigráfico en el litoral noreste de la Hispania citerior", in F. Beltrán (ed.), Roma y el nacimiento de la cultura epigráfica en Occidente, Zaragoza, 97-119.

Mayer, M. (1998), “¿Qué es un Augusteum?, Historia Antiqua 4: 63-70.

Mayer, M. (1999), “Aproximación a la religión cívica en Hispania bajo los 
flavios", Ktema 24: 341-345.

Mayer, M. (2004), "El Augusteum de Narona (Vid, Metković, Croacia) en época de los Severos", in Orbis Antiquus. Studia in honorem Ioannis Pisonis, ClujNapoca, 283-289.

Mayer, M. (2005), "Constantino el Grande: deconstrucción y construcción de un Imperio", in F. de Oliveira (coord.), Génese e Consolidação da Ideia de Europa, vol. III, O Mundo Romano, Coimbra, 203-230.

Mayer, M. (2007a), "La presenza imperiale nelle città del Picenum tra l'epoca augustea e il regno dei Severi : un primo aproccio", Studi Maceratesi 41: 27-40.

Mayer, M. (2007b), "Las dedicatorias a miembros de la domus Augusta julio-claudia y su soporte: una primera aproximación”, in G. Paci (ed.), Contributi all'epigrafia del'età augustea. Actes de la XIII' Rencontre francoitalienne sur l'épigraphie du monde romain, Tivoli, 171-199

Mayer, M. (2008), "Sila y el uso político de la epigrafía", in M. Caldelli, G. L. Gregori, S. Orlandi (eds.), Epigrafia 2006. Atti della XIVerencontre sur l'épigraphie in onore di Silvio Panciera con altri contributi di colleghi, allievi e collaboratori, Roma, 121-135.

Mayer, M. (2009), "Los honores recibidos por la familia de Marco Aurelio en la parte oriental del imperio romano: ¿̨cambio o continuidad en el culto dinástico?", in A. Martínez Fernández (ed.), Estudios de Epigrafía Griega, La Laguna, 277-294.

Mayer, M. (2010), "La presència de la dinastia antonina a Tarraco", in Studia Celtica Classica et Romana Nicolae Szabó septuagesimo dicata, Budapest, 159-167.

Mayer, M. (2015), "La epigrafia y el Augusteum de Narona", in G. Zecchini (ed.), L 'Augusteum di Narona. Atti della Giornata di Studi. Roma 31 maggio 2013, (Centro ricerche e documentazione sull' antichità clàssica, monografie, 3 7), Roma, pp. 19-41.

McCrum, M., Woodhead, A.G. (1961), Select Documents of the Principates of the Flavian Emperors Including the Year of Revolution, A.D. 68-96, Cambridge.

Mednikarova, I. (2003), "The accusative of the name of the deceased in Latin and Greek epitaphs", ZPE 143: 117-134.

Meiggs, R. (1972), The Athenian Empire, Oxford.

Melani, V., Vergari, M. (1985), Profilo di una città etrusca Roselle, Pistoia.

Melchor Gil, E. (1992), "Sistemas de financiación y medios de construcción de la red viaria hispana”, Habis, 23: 121-137.

Melchor Gil, E. (2010), "Homenajes estatutarios e integración de la mujer en la vida pública municipal de las ciudades de la Bética", in F. J. Navarro (ed.), Pluralidad e integración en el Mundo Romano, Pamplona, 221-245. 
Mellor, R. (1975), ӨEA P $\Omega$ MH the Worship of the Goddess Roma in the Greek World, Göttingen.

Mellor, R. (1981), “The Goddess Roma”, in $A N R W$ II 17. 2, Berlin, New York, 950-1030

Menegazzi, L. (1995), Il manifesto italiano (prima ed. 1974), Milano.

Merêa, P. (1929), História de Portugal, Vol. II. Coimbra.

Merêa, P. (1941), Suárez, Grácio, Hobbes, Coimbra.

Mesnard, P. (1977), Essor de la philosophie politique au XVIe Siècle, Paris.

Messerschmidt, W. (2003), Prosopopoiia: Personifikationen politischen Charakters in spätklassischer und hellenistischer Kunst, Köln.

Michelini, A. N. (1994), "Political themes in Euripides' Suppliants", AJPh 115. 2: $219-252$.

Millar, F. (1968), "Local cultures in the Roman Empire: Libyan, Punic and Latin”, JRS 58: 126-134.

Millar, F. (1993), The Roman Near East 31 BC-337 AD, London.

Millar, F. (2006), A Greek Roman Empire, Power and belief under Theodosius II 408-450, Berkeley.

Minerath, R. (1996), Histoire des Conciles, Paris.

$M L H=$ J. Untermann, J. (1975-2000), Monumenta linguarum Hispanicarum, $\mathrm{I}-\mathrm{V}$, Wiesbaden.

Moggi, M. (1976), I sinecismi interstatali greci, Pisa.

Moncada, C. (1947), Filosofia do direito e do estado, I, Coimbra.

Monfrin, J. (1964), "Humanisme et traductions au Moyen Age", in L'Humanisme médieval dans les littératures romanes du XIIe au XIVe siècle (Actes du Colloque organisé par le Centre de Philologie et de Littératures romanes de l'Université de Strasbourg), Paris.

Monteiro, N., d'Encarnação, J. (1993-1994), “A propósito de uma inscrição latina em Santiago da Guarda (Ansião)", Conimbriga 32-33: 303-311.

Moretti, G. (2007), "Patriae trepidantis imago. La personificazione di Roma nella Pharsalia fra ostentum e disseminazione allegorica", Camenae 2: 1-17

Morrison, A. D. (2007), The Narrator in Archaic Greek and Hellenistic Poetry, Cambridge.

Mosley, D. J. (1965), "The Size of Embassies in Ancient Greek Diplomacy”, ТРАРЬA: 255-266.

Mosley, D. J. (1972), "Envoys and diplomacy in Ancient Greece”, Historia 22: 1-97.

Mossé, Cl. (1970), La colonisation dans l'Antiquité, Paris, 27-99. 
Mullen, A. (2007), “Linguistic evidence for 'romanization': continuity and change in Romano-British onomastics: a study of the epigraphic record with particular reference to Bath", Britannia 38: 35-61.

Muñoz, V. (2005), "La interpretatio romana del dios prerromano Bandue", Veleia 22: $145-152$.

Munro, J.A.R. (1926), “Xerxes' Invasion of Greece”, in The Persian Empire and the West, Cambridge Ancient History, Vol. IV, Cambridge, 268-316.

Nascimento, A. A. (1990), "Poggio e o seu interesse por códices de Alcobaça", Revista da Faculdade de Letras de Lisboa 13-14: 37-40.

Nascimento, A. A. (1993), "As librarias dos príncipes de Avis", Biblos. Revista da Faculdade de Letras (Coimbra). Actas do Congreso Comemorativo do $6^{\circ}$ Centenário do Infante D. Pedro (25 a 27 de Novembro de 1992) 69: 265-287.

Nascimento, A. A. (1995), "La réception des auteurs classiques dans l'éspace cultural portugais: une questione ouvert", in C. Leonardi, B. Munk Olsen (eds.), The Classical Tradition in the Middle Ages and Renaissance, Spoleto, 47-56.

Nascimento, A. A. (1997), "Traduzir, verbo de fronteira nos contornos da Idade Média”, in C. Almeida Ribeiro, M. Madureira (eds.), O género do texto medieval, Lisboa, 113-138.

Nascimento, A. A. (1999), Cister. Os documentos primitivos. No 9.० Centenário da fundação de Cister (1999). Introdução, tradução e notas de Aires A. Nascimento, Lisboa.

Navarro Caballero, M. (2003), "Mujer de notable: representación y poder en las ciudades de la España imperial”, in S. Armani, B. Martineau-Hurlet, A. U. Stylow, (eds.), Acta antiqua Complutensia IV. Epigrafía y sociedad en Hispania durante el Alto Imperio: estructuras sociales, Alcalá de Henares, 119-127.

Nemeti, S. (1998), "Cultul lui Sucellus-Dis Pater şi al Nantosueltei-Proserpina în Dacia romană”, EphemNapoc 8: 95-121.

Neumann, G., Untermann, J. (eds.) (1980), Die Sprachen im Römischen Reich der Kaiserzeit. Beihefte der Bonner Jabrbücher 40, Bonn.

Nicosia, F. (ed.) (1990), Un decennio di ricerche a Roselle. Statue e ritratti, Firenze.

Nunes, E., Albuquerque, M. (1968), "Parecer do doutor 'Valasco di Portogallo' sobre o beneplácito régio (Florença, 1954)”, in V. Rau (ed.), Do tempo e da bistoria, Lisboa, t. 2, 97-139.

Ober, J. (1989), Mass and Elite in Democratic Athens. Rhetoric, Ideology, and the Power of the People, Princeton.

Odiot, T. (2004), "Le site du Molard à Donzère", in Brun, J.-P., Poux, M., Tchernia, A. (eds.), Le vin. Nectar des Dieux. Génies des Hommes, Gollion, 202-203. 
Oelmann, F. (1914), Die Keramik des Kastells Niederbieber, Frankfurt.

Ohly, D. (1976), Die Aegineten: die Marmorskulpturen des Tempels der Aphaia auf Aegina. (a) I. Die Ostgiebelgruppe. München. (b) II. Die Westgiebelgruppe. III. Altarplatzgrupen, Akrotere, etc, München.

Olteanu, T. (2008), "El culto a Victoria y la interpretatio indígena en el Occidente de Hispania, Gallia y el norte de Britania", BVallad 74: 197-224.

Ors, A. de (1953), Epigrafia juridica de la España romana, Madrid.

Pacaut, M. (1993), Les moines blancs. Histoire de l'Ordre de Cîteaux, Paris.

Pallottino, M. (1952), "El problema de las relaciones entre Cerdeña e Iberia en la antigüedad prerromana", Ampurias 14: 137-155.

Panciera, S. (2003), "Umano, sovraumano o divino? Le divinità augustee e l'imperatore a Roma", in L. de Blois, P. Erdkamp, O. Hekster, G. De Kleijn, S. Mols, (eds.), The Representation and Perception of Roman Imperial Power. Proceedings of the Third Workshop of the International Network Impact of Empire (Roman Empire c. 200 B.C. - A.D. 476), Amsterdam, 219-239.

Paparelli, G. (1973), Feritas, humanitas, diuinitas. L'essenza umanistica del Rinascimento, Napoli.

Parker, V. (1988), "Túpavvoc. The semantics of a political concept from Archilochus to Aristotle", Hermes 126. 2: 145-172.

Patillon, M. (ed.) (2002), Pseudo-Aelius Aristide, Arts rhétoriques, Paris.

Pekary, T. (1968), Untersuchungen zu den römischen Reichsstraßen, Bona.

Pellegrini, D. P. M. (2003), Le Grandi Storie dell'Auto, vol. 2, Alfa Romeo, 35-35.

Pelling, Ch. (2002), "Speech and action: Herodotus' Debate on the Constitutions", PCPhS 48: 123-158.

Peña Cervantes, Y. (2010), Torcularia. La producción de vino y aceite en Hispania. Catálogo de yacimientos analizados en cedé (Sèrie documenta 149), Tarragona.

Pensa, M. (1979), "Genesi e svilupo dell'arco onorario nella documentazione numismática”, Studi sull'Arco Onorario Romano, Roma, 19-27.

Peres, D. (1952), História de Portugal, II, Porto.

Pérez Martin, A. (1979), Proles Aegidiana. I. Introducción. Los Colegiales desde 1368 a 1500 , Bolonia.

Pérez Martin, A. (1999), Españoles en el Alma Mater Studiorum. Profesores hispanos en Bolonia (de fines del siglo XII a 1799), Murcia.

Pérez Ruiz, F. (1984), "El justo es feliz y el injusto desgraciado, justicia y felicidad en la República de Platon", Pensamiento 40, 159: 257-295.

Petrarca, F. (1581), Francisci Petrarchae Florentini Opera. Basileae, per Sebastianum Henricpetri. 
Petrarca, F. (1942), Epistolae familiares, in V. Rossi (ed.), Le Familiari, Firenze.

Petri, Ch. (1989), 'La politique de Constance II: un premier 'césaropapisme' ou l'imitatio Constantini?", in A. Dihle (coord.), L'église et l'empire au IV siècle, Genève, 113-178.

Pfeiffer, R. (1949-1951), Callimachus, 2 vols., Oxford.

Pflaum, H.G. (1976), Inscriptions latines de l'Algérie, t. II, vol. II, Inscriptions de la Confédération cirtéenne, de Cuicul et de la tribu des Suburbures, Alger.

Pflug, H. (1941), As auto-estradas do Reich, Berlim.

Pharr, C. et alii (2008), The Theodosian Code and Novels and the Sirmondian Constitution. Translation, commentary and bibliography, Union (NJ).

Piana, C. (1976), Nuovi documenti sull'Universitá di Bologna e sul Collegio di Spagna,I-II, Bolonia, Zaragoza.

Pick, B. (1898), Die antiken Münzen Nordgriechenlands I, 2. Die antiken Münzen von Dacien und Moesien, Berlin.

Piganiol, A. (1972, 2a ed.), L'empire chrétien, Paris.

Pina, R. de (1977), Chronica do Senhor Rey D. Affonso V, cap. CXXV "Das feiçoões custumes e virtudes do Yfante Don Pedro”, in M. L. de Almeida (Intro. e Revisão), Crónicas de Rui de Pina, Porto.

Pinheiro Futre, M. P. (2006), "Do Mito à Utopia: viagem ao mundo do imaginário grego" in Actas do V Congresso da APEC-Antiguidade Clássica e nós: Herança e Identidade Cultural, Braga, 569-581.

Pinho, S. T. (1999), "Os Príncipes de Avis e o Pré- Humanismo Português", in Raizes Greco-Latinas da Cultura Portuguesa. Actas do I Congresso da APEC, Coimbra, 99-133.

Pinto, Frei H. (1952), "Diálogo da justiça”, in Imagem da vida cristã, I, Lisboa.

Pippidi, D. M. (1971), I Greci nel Basso Danubio dall'età arcaica alla conquista romana, Mailand.

Pirling, R. (1993), "Ein Trierer Spruchbecher mit ungewöhnlicher Inschrift aus Krefeld-Gellep", Germania 71: 387-404.

Podlecki, A. J. (1976), “Athens and Aegina”, Historia 25.4: 396-413.

Poenaru Bordea, G. (1979), "Les statères ouest-pontiques de type Alexandre le Grand et Lysimaque”, RBNS 125: 37-51.

Prag, J. R. W. (2002), "Epigraphy by numbers: Latin and the epigraphic culture in Sicily", in A. E. Cooley (ed.), Becoming Roman, Writing Latin? Literacy and Epigraphy in the Roman West. JRA Suppl. Ser. 48: 15-31.

Preda, C., Popescu, E., Diaconu, P. (1962), "Săpăturile arheologice de la Mangalia (Callatis)", Materiale 8: 439-455.

Pressouyre, L. (1990), Le rêve cistercien, Paris. 
Price, S. R. F. (1984), Rituals and Power. The Roman Imperial Cult in Asia Minor, Cambridge.

Privitera, G. A. (1988), "Pindaro, Nem. III 1-5 e l'acqua di Egina”, QUCC 58: 63-70.

Puerta Torres, C. (1995), Los miliarios de la Vía de la Plata, 1-2, Madrid.

Quadrino, D. (2007), Una nuova iscrizione onoraria di Adriano e il Sebasteion di Kestros in Cilicia Tracheia, Tivoli.

Radnoti Alföldi, M., Rasbach, G. (1999), "Zur Frage der interpretatio Romana“, in Festschrift für Günter Smolla, Wiesbaden, 597-605.

Raepsaet-Charlier, M. Th. (1975), "La datation des inscriptions latines dans les provinces occidentales de l'Empire Romain d'après les formules " In $\mathrm{H}$ (onorem) D(omus) D(ivinae) » et «Deo, Deae »", in ANRW II 3: 232-282.

Raepsaet-Charlier, M. Th. (2005), "Les sacerdoces des femmes sénatoriales sous le Haut-Empire", in M.-F. Baslez, F. Prévot (eds.), Prosopographie et histoire religieuse. Actes du colloque tenu en l'Université Paris XII-Val de Marne le 27 E 28 octobre 2000, Paris, 283-304.

Ramalho, A. C. (1985), Latim Renascentista em Portugal (Antologia), Coimbra.

Rapp, Cl. (2005), Holy Bishops in Late Antiquity, The nature of Christian Leadership in an age of transition, Berkeley.

Rau, V. (1969), "Italianismo na cultura jurídica portuguesa do século XV", Revista Portuguesa de História 12.1: 185-206.

Rau, V. (1973), "Studenti ed eruditi portoghesi in Italia nel secolo XV", Estudos Italianos em Portugal 36: 7-73.

Rawlinson, H. G. (1916), Intercourse between India and the Western World from the Earliest Times to the Fall of Rome, Cambridge.

Rebelo, D. L. (1951), Do governo da republica pelo rei (de republica gubernanda per regem), reprodução fac-similada da edição de 1496, Introdução e notas de A. M. de Sá, Lisboa.

Rebelo, L. de S. (1983), A concep̧̣ão do poder em Fernão Lopes, Lisboa.

Rebuffat, R. (2007), "Pour un corpus des bilingues punico-libyques et latinolibyques", in M. H. Fantar (ed.), Osmose etnho-culturelle en Méditerranée, Tunis, 183-242.

Regra do Patriarca S. Bento (1992), Edições “Ora \& Labora”, Singeverga.

Rhodes, P. J. (1993), A Commentary on the Aristotelian ATHENAION POLITEIA, Oxford.

Rhodes, P. J. (2006), A History of the Classical Greek World 478-323 BC, Molden. RIB = Collingwood, R. G. (1965), The Roman inscriptions of Britain. I. Inscriptions on stone, Oxford. 
RIG = P.-M. Duval (ed.), Recueil des inscriptions gauloises, Paris 1985-. I: M. Lejeune, Textes gallo-grecs, 1985; II.1: M. Lejeune, Textes gallo-étrusques. Textes gallo-latins sur pierre, 1988; II.2: P.-Y. Lambert, Textes gallo-latins sur instrumentum, 2002; III: P.-M. Duval y G. Pinault, Les calendriers (Coligny, Villards d'Héria), 1988; IV: J.-B. Colbert de Beaulieu y B. Fischer, Les légendes monétaires, 1998.

Ripollés, P. P. (2004), "Coinage and identity in the Roman provinces: Spain", in Ch. Howgego, V. Heuchert, A. Burnett (eds.), Coinage and identity in the Roman provinces, Oxford, 79-93.

Ripollés, P. P., Velaza, J. (2002), "Saguntum, colonia latina”, ZPE 141: 285-294.

Rodgers, B. (1989), “The Metamorphosis of Constantine”, CQ 39.1: 233-246.

Rodrigues, M. A. (1993), "O infante D. Pedro e a Universidade”, Biblos. Revista de Faculdade de Letras (Coimbra). Actas do Congreso Comemorativo do $6^{\circ}$ Centenario do Infante D. Pedro (25 a 7 de Novembro de 1992) 69: 345362.

Rodrigues, N. S. (2007), "Entre Europa e Io: elementos orientais na arte grega arcaica e clássica", in J. A. Ramos, L. M. Araújo, A. Ramos dos Santos (eds.), Arte Pré-Clássica. Colóquio Comemorativo dos Vinte Anos do Instituto Oriental da Faculdade de Letras da Universidade de Lisboa, Lisboa, 323346.

Rodríguez, P., Díez de Pinos, E. (2014), "Nueva inscripción celtibérica en piedra de E1 Pueyo de Belchite (Zaragoza)", Palaeohispanica 14: 245-262.

Rodríguez Colmenero, A., Ferrer Sierra, S., Álvarez Asorey, R. (2004), Miliários e outras inscricións viarias romanas do noroeste hispánico. Santiago de Compostela.

Rocha Pereira, M. H. (1981), "O mais antigo texto europeu de teoria política", Nova Renascença 1: 364-370.

Rocha Pereira, M. H. (1990), “O 'Diálogo dos Persas’ em Heródoto”, Estudos Portugueses. Homenagem a António José Saraiva, Lisboa, 351-362.

Rocha Pereira, M. H. (2003), Hélade. Antologia da Cultura Grega, Asa, Porto.

Rocha Pereira, M. H. (2008, 8a ed.), Sófocles: Antígona, Coimbra.

Roha Pereira, M. H. (2009, 10ª ed.), Hélade, Lisboa, Guimarães.

Rocha Pereira, M. H. (2012), Estudos de História da Cultura Clássica, vol.1 Cultura Grega, Lisboa.

Roldán Hervás, J. (1975), Itineraria Hispana. Fuentes antiguas para el estudio de las vías romanas en la Peninsula Ibérica, Madrid.

Röllig, W. (1980), “Das Punische im Römischen Reich”, in G. Neumann, J. Untermann (eds.), Die Sprachen im Römischen Reich der Kaiserzeit. (Bonner Jabrbücher des Rheinischen Landesmuseums in Bonn im Landschaftsverband 
Bibliografia

Rheinland und des Vereins von Altertumsfreunden im Rheinlande 40), Köln, 285-299.

Romano, E. (2006-2009), "Le tombe "a cupa" in Italia e nel Mediterraneo. Tipologia architettonica, committenza e rituale”, StClOr 52: 149-219.

Romilly, J. de (1959), "Le classement des constitutions d'Hérodote à Aristote", REG 72: 81-99.

Rose, C. B. (1997), Dynastic Commemoration and Imperial Portraiture in the Julio-Claudian Period, Cambridge.

Rosenthal, F. (1936), Die Sprache der palmyrenischen Inschriften und ibre Stellung innerhalb des Aramäischen, Leipzig.

Rosivach, V. J. (1977), "Earthborns and Olympians: the parodos of the Ion", CQ 27. 2: 284-294.

Rosivach, V. J. (1988), "The Tyrant in Athenian Democracy", QUCC 59: 43-57.

Rossillon, Ph. (ed.) (1995), Atlas de la langue française, Paris.

Rossiter, J. J. (1978), Roman Farm Buildings in Italy (BAR int. Ser. 52), Oxford.

Rössler, O. (1980), "Libyen von der Cyrenaica bis zur Mauretania Tingitana", in G. Neumann, J. Untermann (eds.), Die Sprachen im Römischen Reich der Kaiserzeit. (Bonner Jabrbücher des Rheinischen Landesmuseums in Bonn im Landschaftsverband Rheinland und des Vereins von Altertumsfreunden im Rheinlande 40). Köln, 267-284.

Rubenstein, L. (2004), "Ionia", in M. H Hansen, T. H. Nielsen (eds.), An Inventory of Archaic and Classical poleis, Oxford, 1053-1107.

Rucquoi, A. (2003), "Rois et princes portugais chez les auteurs castillans du $\mathrm{XV}^{\mathrm{e} m e}$ siécle», Península. Revista de Estudos Ibéricos. Entre Portugal e Espanha. Relaçôes Culturais (sécolos XV-XVIII). In Honorem Jose Adriano de Freitas Carvalho, 0: 39-51.

Ruggini, L. C. (1989), "Felix Temporum Reparatio", in A. Dihle (coord.), Realtà socio-economiche in movimento durante un ventennio di regno (Costanzo II Augusto, 337-361 d.C.), L'église et l'empire au IV siècle, Genève, 179-243.

Rüpke, J. (2005), Fasti sacerdotum. Die Mitglieder der Priesterchaften und das sakrale Funktionspersonal römischer, griechischer, orientalischer und jüdischchristlicher Kulte in der Stadt Rom von 300 v. Chr. bis 499 n. Chr., Wiesbaden.

Rusjaeva, A., Vinogradov, Ju. G., (2000), "Apollon Ietros. Herrscher von Istros”, in A. Avram, M. Babeş (eds.), Olbia, Civilisation grecque et cultures antiques périphériques. Hommages à P. Alexandrescu à son $70^{\circ}$ anniversaire, Bucarest, 229-234.

Rutishauer, B. (2012), Athens and the Cyclades. Economic Strategies 540-314 BC, Oxford. Sabbadini, R. (1905), Le scoperte dei codici latini e greci ne' secoli XIV e XV , Florencia. 
Sabbadini, R. (1914), Le scoperte dei codici latini e greci ne' secoli XIV e XV, Florencia.

Saddington, D.B. (1999), "Roman soldiers, local gods and interpretatio Romana in Roman Germany", ActaCl 42:155-169.

Salazar, A. M. (1976), "El impacto humanístico de las misiones diplomáticas de Alonso de Cartagena en la Corte de Portugal entre medievo y renacimiento (1421-31)", in A. D. Deyermond (ed.), Medieval Hispanic Studies presented to Rita Hamilton, Londres, 215-226.

Salinas, M. (1995), "Los inicios de la epigrafía en Lusitania oriental", in F. Beltrán (ed.), Roma y el naámiento de la cultura epigráfica en Occidente, Zaragoça, 281-291.

Salway, B. (2001), "Travel, Itineraria and Tabellaria", in C. Adams and R. Laurence (eds.), Travel and Geography in the Roman Empire, Londres, Nova Iorque, 22-66.

Santo Agostinho (2009 12a ed. ), A cidade de Deus, trad. de Oscar Paes Leme, 2 v., Vozes, Petrópolis, São Paulo.

Santos, M. J. A. (1998), Vida e morte de um mosteiro cisterciense. S. Paulo de Almaziva - Séculos XIII-XV, Lisboa.

Saumagne, C. (1928), "Iter populo debetur", Révue d'Histoire, de Littérature et d'Histoire Anciennes 54: 320-353.

Scheer, T. S. (2003), "The Past in na Hellenistic Present: Myth and Local Tradition", in A. Erskine (ed.), A Companion to the Hellenistic World, Oxford, 216-231.

Scheid, J. (2015), "Les Augustea et le culte des empereurs. Réflexions sur les rites célébrés dans ces lieux de culte", in P. Gros, E. Marin, M. Zink (eds.), Auguste, son époque et l 'Augusteum de Narona. Actes du colloque organisé à l'Académie des Inscriptions et Belles-Letres et l'Université Catholique de Croatie (Zagreb) 12 décembre 2014, 17-30, Paris.

Schilardi, G. (ed.) (1997), Filostrato. Immagini, Lecce.

Schmidt, R. (1980), "Die Ostgrenze von Armenien über Mesopotamien, Syrien bis Arabien”, in G. Neumann, J. Untermann (eds.), Die Sprachen im Römischen Reich der Kaiserzeit. (Bonner Jahrbücher des Rheinischen Landesmuseums in Bonn im Landschaftsverband Rheinland und des Vereins von Altertumsfreunden im Rheinlande 40). Köln, 187-214.

Schmidt, Th., Fleury, P. (2011), Perceptions of the Second Sophistic and its Times. Regards sur la seconde sophistique et son époque, Toronto, Buffalo, London.

Schwartz, J. (1960), Pseudo-Hesiodeia: recherches sur la composition, la diffusion et la disparition ancienne d'oeuvres attribuées à Hésiode, Leiden.

Scott, K. (1936), The Imperial Cult under the Flavians, Stuttgart.

Sealey, R. (1976), A bistory of Greek city-states 700 -338 B. C. Berkeley. 
Seignobos, Ch. (1969), Histoire sincère de la nation française, Paris.

Semerari, L. (2000), Aula Magna Università degli Studi di Bari, Bari.

Sergent, B. (2006), "Sucellus et le tonneau", in Anthropology of the Indo-European World and Material Culture. Proceedings of the 5th International Colloquium of Anthropology of the Indo-European World and Comparative Mythology, Budapest, 61-80.

Serra, J. C. da (1972), Academia Real das Sciencias de Lisboa, II, cap. VII, Lisboa. Sforza, W. C. (1951), “Osservazioni sul 'De nobilitate legum' di Coluccio Salutati”, in E. Castelli (ed.), Umanesimo e Scienza politica (Atti del congresso Internazionale di Studi Umanistici, Roma-Firenze, 1949), Milano.

Shapiro, H.A. (1993), Personification in Greek art: the representation of abstract concepts 600-400 b.C., Zürich.

Shaw, M. H. (1982), “The $\tilde{\eta} \theta$ os of Theseus in 'The Suppliant Women”, Hermes 110. 1: 3-19.

Shorrock, R. (2011), The Myth of Paganism: Nonnus, Dionysus and the World of Late Antiquity, Bristol.

Sigeia, L. (1970), Dialogue de deux jeunes filles sur la vie de retraite (1552), Présenté, traduit et annoté par O. Sauvage (ed.), Paris.

Sillières, P. (1990), Les voies de communication de l'Hispanie méridionale, Paris.

Silva, N. J. E. G. (1964), Humanismo e Direito em Portugal no século XVI, Lisboa.

Simón, I. (2013), Los soportes de la epigrafía paleohispánica. Inscripciones sobre piedra, bronce y cerámica, Zaragoza, Sevilla.

Siniscalco, P. (2004, 5a ed.), Il cammino di Cristo nell'Impero romano, Roma, Bari.

Slavazzi, F. (2006), "Il ciclo di relievi della Kaisersaal del ginnasio di Vedio a Efeso", in Iconografía 2005. Immagini e immaginari dell'antichità classica al mondo moderno, Roma, 235-243

Smyth, A. C. (2011), Polis and Personification in Classical Athenian Art, Leiden.

Snodgrass, A. M. (1977), Archaeology and the rise of the Greek state, Cambridge.

Snodgrass, A. M. (1980), Archaic Greece. The age of experiment, Londres.

Soares, C. (2008), Platão. O Político. Tradução do grego, introdução e notas, Lisboa.

Soares, C. (2014), “Theoria e práxis política em Heródoto”, Cuadernos de Filología Clássica: Estudios griegos e indoeuropeus 24: 57-79.

Soares, N. C. (1994), O príncipe ideal no século XVI e a obra de D. Jerónimo Osório, Coimbra.

Soares, N. C. (2002), "O infante D. Pedro e a cultura portuguesa", Biblos. Revista da Faculdade de Letras 78:107-128. 
Sodano, A. R. (1970), Porphyrii Quaestionum Homericarum Liber I, Napoli.

Solas, J. G. (2008), “Escrito sobre la ciudad”, Pensar la publicidad, II, n. 2: 37-62.

Sordi, M. (1965), Il cristianesino e Roma, Bologna.

Sordi, M. (1984), I cristiani e l'impero romano, Milano.

Soria, A. (1956), Los humanistas de la Corte de Alfonso el Magnánimo (según los epistolarios), Granada.

Sousa, D. A. C. de (1946-1954), Memória dos livros do uso del Rey D. Duarte, in Provas da história genealógica da casa real portuguesa, tomo I, liv. III, Coimbra.

Sousa, R., Fialho, M. C., Haggag, M., Rodrigues, N. S. (2013), Alexandrea ad Aegyptum: The Legacy of Multiculturalism in Antiquity, Lisboa.

Spickermann, W. (1997), "Aspekte einer neuen regionalen Religion und der Prozess der "interpretatio" im römischen Germanien, Rätien und Noricum", in Römische Reichsreligion und Provinzialreligion, Tübingen, 145-167.

Spyridakis, S. (1968), “Zeus is Dead: Euhemerus and Crete”, CJ 63: 337-340.

Stafford, E., Herrin, J. (eds.) (2005), Personification in the Greek World from Antiquity to Byzantium, Burlington.

Statuta capitulorum generalium ordinis Cisterciensis ab anno 1116 ad annum 1786 edidit Josephus M. ${ }^{\text {ia } C a n i v e z ~(1933-1941), ~} 8$ vols., Louvain.

Stefan, A. (2005), "Le titre de filius Augustorum de Maximin et Constantin et la théologie de la tétrarchie", in M.-F. Baslez, F. Prévot (eds.), Prosopographie et histoire religieuse. Actes du colloque tenu en l'Université Paris XII-Val de Marne le 27 Eं 28 octobre 2000, Paris, 329-349

Stefani, G. (1986), "I cippi a botte della provincia Sardinia”, Nuovo bullettino Archeologico Sardo 3: 115-160.

Stefani, G. (1988), "Cippi a botte nella basilica di S. Saturnino a Cagliari”, Quaderni della Soprintendenza archeologica per le province di Cagliari e Oristano 5: 167-175.

Stegmann, A. (1977), "La place de la praxis dans la notion de 'raison d'État' ", in Théorie et pratique politiques à la Renaissance, Paris.

Steinbrecher, M. (1985), Der Delisch-Attischen Seebund und die AthenischSpartanischen Beziehungen in der Kimonischen Ära (478/77-462/1), Berlin.

Stemmer, K (ed.) (1995), Standorte - Kontext und Funktion antiker Skulptur, Berlin.

Sterckx, C. (2008), "Sucellos et le casque d'Hadès", in Philomythia. Mélanges offerts à Alain Moreau, Monts, 223-229.

Stern, J. (1996), Palaephatus. Peri Apiston: On Unbelievable Tales, Wauconda. 
Stern, J. (1999), "Rationalizing Myth: Methods and Motives in Palaephatus" in R. Buxton, R. (ed.), From Myth to Reason? Studies in the Development of Greek Thought, Oxford, 215-222.

Stewart, A. (1990), Greek Sculpture: an exploration, New Haven, Yale.

Storey, I. C. (2003), Eupolis poet of old comedy, Oxford.

Stowe Mead, G. R. (1901), Apollonius of Tyana, the Philosopher-Reformer of the First Century A.D., London.

Strassler, R. B. (ed.) (2007), Landmark Herodotus: The Histories, New York.

Strassler, R.B. (ed.) (2009), Landmark Herodotus: The Histories, New York.

Strootman, R. (2010), "Literature and the Kings", in Clauss, J., Cuypers, M. (eds.), A Companion to Hellenistic Literature, Malden, Oxford, 30-45.

Suberbiola Martínez, J. (1987), Nuevos concilios hispano-romanos de los siglos III y IV. La colección de Elvira, Málaga.

Szabó, Á. (2007), Daciai papság, Budapest.

Szabó, Á. (2008), "Sulla questione dello statuto giuridico dei sacerdoti provinciali durante il principato. Studio preliminare”, Iustum Aequum Salutare 4: 71-81.

Tamerl, I. (2008), Das Holzfass in der römischen Antike mit einer Studie zu Fassfunden in Raetien, Diplomarbeit presso l'Università di Innsbruck, consultabile presso la Universitäts- und Landesbibliothek Innsbruck DG43696.

Tate, J. (1927), “The Beginnings of Greek Allegory”, CR 41.6: 214-215.

Tchernia, A. (1986), Le vin de l'Italie romaine. Essai d'bistoire économique d'après les amphores (BEFAR 261), Rome.

Teive, D. de (1786), Epodos Que Cont'em Sentenças Uteis A todos os Homens, A's quaes se acrescentão Regras para a boa educação de hum principe. Trad. no vulgar em verso solto por Francisco de Andrade (conforme à ed. de Lisboa, 1565), Lisboa, Na Of. Patr. de Francisco Luiz Ameno.

Temporini, H. (1978), Die Frauen am Hofe Trajans. Ein Beitrag zur Stellung der Augustae im Principat, Berlin, New York.

Thomson de Grummond, N. (2006), Etruscan Myth. Sacred History, and Legend, Philadelphia.

Tomlin, R. S. O. (1987), "Was ancient British Celtic ever a written language? Two texts from Roman Bath", Bulletin of the Board of Celtic Studies 34: 18-25.

Topál,J. (1990), “Der Import der sogenannten Moselweinkeramik in Pannonien”, ReiCretActa 27-28: 177-184.

Tortorici, E. (1975), Castra Albana. Forma Italia, Regio I, Roma.

Touchard, J. (1959), Histoire des idées politiques, I. Paris [trad. port. Lisboa, 1970]. 
Tranoy, A. (1981), La Galice romaine, Paris.

Tuchelt, K. (1981), "Zum Problem Kaisareion-Sebasteion. Eine Frage zu den Anfängen des römischen Kaiserkultes”, MDAI, 31 : 167-186.

Ulbert, G. (1959), "Römische Holzfässer aus Regensburg”, Bayerische Vorgeschichtsblätter 24: 6-29.

Ullman, B. L. (1963), The humanism of Coluccio Salutati, Padova.

Ullmann, W. (1980), Radici del Rinascimento (tr. ital.), Roma, Bari.

Unz, R.K. (1985), "The Surplus of the Athenian phoros", GRBS 26: 21-42.

Ureña Prieto, M. H. (2001), Dicionário de Literatura Grega, Lisboa.

Valiño, A. (1999), "La cerveza en las fuentes romanas. Base textual y fijación de su importancia”, AncHistB 13: 60-71.

Van Haeperen, F. (2002), "Le collège pontifical (3ème s. a.C.-4ème s. p.C.)", Études de Philologie, d'Archéologie et d'Histoire Anciennes 39: 11-42.

Varner, E.R. (2004), Mutilation and transformation. Damnatio memoriae and Roman Imperial Portraiture, Leiden, Boston.

Várzeas, M. I. O. (2013), "Callimachus and the New Paths of Myth”, in R. Sousa et alii (coord.) Alexandrea ad Aegyptom: the legacy of multiculturalismo in antiquity. Lisboa.

Velaza, J. (2003), "Epigrafía ibérica emporitana: bases para una reconsideración”, Palaeohispanica 3: 179-192.

Velaza, J. (2003a), "Las inscripciones monetales”, in P. P. Ripollés, M. del M. Llorens, Arse-Saguntum. Historia monetaria de la ciudad y su territorio, Sagunto, 121-148.

Velaza, J. (2009), “Epigrafía y literacy paleohispánica en territorio vascón”, Palaeohispanica 9: 611-622.

Vergerio, P. P. (1934), “Epistolario di Pier Paolo Vergerio”, in L. Smith (ed.), Fonti per la storia d'Italia, vol. 74, Roma, 436-445.

Vierneisel, K., Zanker, P. (1979), Die Bildnisse des Augustus: Herrscherbild und Politik in kaiserlichen Rom, München.

Villar, F., Pedrero, R. (2001), “Arroyo de la Luz III”, Palaeohispanica 1: 235274.

Vinogradov, J. G. (2000), "Heilkundige Eleaten in den Schwarzmeergründungen”, in M. Dreher (ed.), Bürgersinn und staatliche Macht. Festschrift für Wolfgang Schuller zum 65. Geburtstag, Konstanz, 133-149.

Vittinghoff, F. (1951), Römische Kolonisation und Bürgerrechtspolitik unter Caesar und Augustus, Wiesbaden.

Vives, J., Marín, T., Martínez, G. (1963), Concilios visigóticos e hispano-romanos, Madrid, Barcelona. 
Voragine, T. (2004), Legenda Aurea. Apresentação do Cardeal Dom José Saraiva Martins e introdução do Doutor Aníbal Pinto de Castro. Tomo Segundo, Porto.

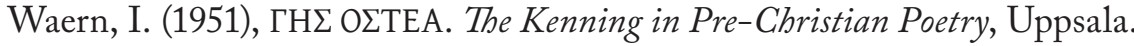

Wallace, M. B., Figueira, T. J. (2010), "Notes on the Island Phoros", ZPE 172: 65-69.

Wallace-Hadrill, A. (2005), "Mutatas formas: The Augustan Transformation of Roman Knowledge", in K. Galinsky (ed.), The Cambridge Companion to the Age of Augustus, Cambridge, 55-84.

Wallinga, H. T. (2005), Xerxes' Greek Adventure. The Naval Perspective, Leiden.

Walter, H. (1993), Ägina: die archäologische Geschichte einer griechischen Insel, München.

Walters, K. R. (1981), "Four Hundred Athenian Ships at Salamis?”, RbM 124: 199-203.

Wankel, H. (1983), "Thukydides 1,74,1 und die Schiffszahlen von Salamis," ZPE 52: 63-66.

Wells, J. (1923), Studies in Herodotus, Oxford.

Wesseling, P. (ed.) (1735), “Itinerarium Antonini Augusti”, Vetera Romanorum Itineraria, Amesterdão.

West, M. L. (1985), The Hesiodic Catalogue of Women: Its Nature, Structure, and Origins, Oxford.

Westrem, S. D. (2001), The Hereford Map. A Transcription and Translation of the Legend with Commentary, Turnhout.

Williams, D. (1987), "Aegina, Aphaia-Tempel XI: the pottery from the second limestone temple and the later history of the sanctuary", $A A$ : 629-680.

Williamson, G. (2004), "Aspects of identity", in C. Howgego, V. Heuchert, A. Burnett (eds.), Coinage and Identity in the Roman Provinces, Oxford, 19-27.

Winiarczyk, M. (2013), The «Sacred History» of Eubemerus of Messene, Berlin.

Witschel, Chr. (1995a), "Römische Tempelkultbilder und Römische Kaiserstatuen als Tempelkultbilder", in K. Stemmer, (ed.), Standorte. Kontext und Funktion antiker Skulptur; Ausstellungskatalog Abgußsammlung, Berlin, 250-265.

Witschel, Chr. (1995b), "Statuen auf römischen Platzanlagen unter besonderer Berücksichtigung von Timgad (Algerien)", in K. Stemmer (ed.), Standorte. Kontext und Funktion antiker Skulptur; Ausstellungskatalog Abgußsammlung, Berlin, 332-358.

Witschel, Chr. (2002), "Zum Problem der Identifizierung von munizipalen Kaiserkultstätten”, Klio 84: 114-124. 
Wlosok, A. (ed.) (1978), Römischer Kaiserkult, Darmstadt.

Wojciechowski, P. (2002), "Il culto di Beleno ad Aquileia romana. Origini, interpretatio Romana e la cosiddetta rinascita celtica", in Gli echi della terra. Presenze celtiche in Friuli. Dati materiali e momenti dell' immaginario. Convegno di studi, Castello di Gorizia, 5 - 7 ottobre 2001, Pisa, 29-35.

Woodard, R. (ed.) (2007), The Cambridge Companion to Greek Mythology, Cambridge.

Woodhead, A. G. (1962), The Greeks in the West. London. (Trad. port., Os Gregos no Ocidente).

Woolf, G. (1996), "Monumental writing and the expansion of the Roman society in the Early Empire", JRS 86: 22-39.

Woolf, G. (2002), "Afterword. How the Latin West was won”, in A. Cookey (ed.), Becoming Roman, writing Latin? Literacy and Epigraphy in the Roman West, JRA Suppl. Ser. 48: 181-188.

Yatromanolakis, Y. (2005), "Poleos erastes. The Greek city as the beloved", in E. Stafford, J. Herrin (eds.), Personification in the Greek World: From Antiquity to Byzantium, London, 267-284.

Young, T. Cuyler (1980), “480/479 B.C. - A Persian Perspective”, Iranica Antiqua 15: 213-39.

Zamora, J. A. (2005), "La práctica de escribir entre los primeros fenicios peninsulares y la introducción de la escritura entre los pueblos paleohispánicos”, Palaeohispanica 5: 155-19.

Zanichelli, G. Z. (2005), "Il mito di Troia nell'immaginario medievale”, in G. Burzacchini (coord.), Troia tra realtà e leggenda, Parma.

Zanker, P. (1983), Provinzielle Kaiserporträts. Zur Rezeption der Selbstdarstellung der Princeps, München.

Zaoli, G. (1912), “Lo Studio bolognese e papa Martino V”, Studi e Memorie per la storia dell'Università di Bologna I - série v. III: 105-188.

Zecchini, G. (ed.) (2015), L'Augusteum di Narona. Atti della Giornata di Studi. Roma 31 maggio 2013, (Centro ricerche e documentazione sull'antichità clàssica, monografie, 37), Roma.

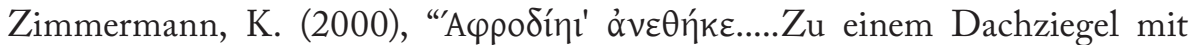
Votivinschrift", in A. Avram, M. Babeş (eds), Olbia, Civilisation grecque et cultures antiques périphériques. Hommages à $P$. Alexandrescu à son $70^{\circ}$ anniversaire, Bucarest, 239-251.

Zurara, G. E. de (1972), Chronica do Conde Dom Pedro de Meneses, II, Lisboa. 